\title{
Improving the Measurement of Shared Cultural Schemas with Correlational Class Analysis: Theory and Method
}

\author{
Andrei Boutyline \\ University of California, Berkeley
}

\begin{abstract}
Measurement of shared cultural schemas is a central methodological challenge for the sociology of culture. Relational Class Analysis (RCA) is a recently developed technique for identifying such schemas in survey data. However, existing work lacks a clear definition of such schemas, which leaves RCA's accuracy largely unknown. Here, I build on the theoretical intuitions behind RCA to arrive at this definition. I demonstrate that shared schemas should result in linear dependencies between survey rows-the relationship usually measured with Pearson's correlation. I thus modify RCA into a "Correlational Class Analysis" (CCA). When I compare the methods using a broad set of simulations, results show that CCA is reliably more accurate at detecting shared schemas than RCA, even in scenarios that substantially violate CCA's assumptions. I find no evidence of theoretical settings where RCA is more accurate. I then revisit a previous RCA analysis of the $1993 \mathrm{General}$ Social Survey musical tastes module. Whereas RCA partitioned these data into three schematic classes, CCA partitions them into four. I compare these results with a multiple-groups analysis in structural equation modeling and find that CCA's partition yields greatly improved model fit over RCA. I conclude with a parsimonious framework for future work.
\end{abstract}

Keywords: cultural schemas; culture and cognition; heterogeneous logics; music tastes; omnivorousness; network analysis

Citation: Boutyline, Andrei. 2017. "Improving the Measurement of Shared Cultural Schemas with Correlational Class Analysis: Theory and Method." Sociological Science 4: 353-393.

Received: July 22, 2016

Accepted: April 4, 2017

Published: May 29, 2017

Editor(s): Olav Sorenson, Gabriel Rossman

Dol: $10.15195 / \mathrm{v} 4 . a 15$

Copyright: (C) 2017 The Author(s). This open-access article has been published under a Creative Commons Attribution License, which allows unrestricted use, distribution and reproduction, in any form, as long as the original author and source have been credited. (0)(1)
THE task of revealing intelligible structures of meaning beneath complex collec1 tions of cultural data is among the most central methodological challenges posed by the sociology of culture (Mohr 1998; Mohr and Rawlings 2012). From the perspective of culture and cognition, this task is a search for shared "cultural schemas" - abstract cognitive structures that specify relationships between cultural elements. In a high-profile recent work, Goldberg (2011) proposed an innovative methodology for identifying groups of survey respondents who share such cultural schemas, which he terms Relational Class Analysis (RCA). RCA has rightfully garnered a substantial amount of attention across diverse domains of study, including cultural tastes (Goldberg 2011; Daenekindt 2017), public opinion (Baldassarri and Goldberg 2014; Wu 2014), organizational behavior (Miranda, Summers, and Kim 2012), and economic sociology (DiMaggio and Goldberg 2010). However, existing work has not yet provided a clear definition of shared cultural schemas, the central concept under investigation. This is a crucial limitation because, without such a definition, RCA's accuracy in locating such schemas cannot be convincingly demonstrated.

Goldberg (2011) introduced RCA using a survey of musical tastes as his case study. Taking a cue from relational theories of meaning (e.g., de Saussure [1916] 
2013; Lévi-Strauss 1963), RCA searches the data for schemas that define not the musical tastes themselves but the relationships between these tastes-that is, which genre tastes are perceived as similar and which are perceived as opposed. This kind of schema can be found in the implicit agreement between an individual who likes musical genres $\mathrm{A}$ and $\mathrm{B}$ but dislikes genre $\mathrm{C}$, and another who dislikes $\mathrm{A}$ and $\mathrm{B}$ but likes $C$ : though the two hold no tastes in common, they nonetheless agree that $A$ "goes with" B, whereas $C$ is "opposed to" $A$ and B. On the other hand, an individual who likes all three of the genres $(\mathrm{A}, \mathrm{B}$, and $\mathrm{C})$ has two tastes in common with the first individual but does not agree that $\mathrm{C}$ is the opposite of $\mathrm{A}$ and $\mathrm{B}$. Thus, under this conception, the first two individuals arrange their tastes according to the same cultural schema, whereas the third one does not. ${ }^{1}$ The goal of RCA is to partition the survey population into classes of respondents who share such cultural schemas-a novel methodological task which is in itself a bold conceptual innovation.

At the core of RCA's approach is a novel similarity measure termed "relationality." Goldberg contends that relationality can quantify the extent to which two respondents organize their attitudes according to a shared cultural schema. However, he does not provide a clear definition of such shared schemas. With this key link between theory and measurement missing, relationality's ability to successfully capture such schemas cannot be convincingly shown. In this article, I examine the theoretical intuitions implicit in current work to arrive at this missing definition. I demonstrate that in order to detect shared cultural schemas like those in Goldberg's (2011:1404-1405) motivating example, relationality must measure the degree of linear dependency between two individuals' vectors of responses. This lends itself to a simple, intuitively plausible formal model of schematic similarity as linear dependence between response vectors, and it suggests that Pearson's correlation may already provide a solution for the task that relationality sets out to solve. When I reexamine Goldberg's motivating example with correlation, I find that it indeed yields more accurate results than relationality. Its results match Goldberg's own description of the data, whereas relationality's do not.

I then used simulations to verify that this difference in accuracy generalizes more broadly. First, I simulated 10,000 test cases in which shared schemas resulted in linear dependencies between responses, as they do in my model and in Goldberg's example. I analyzed each simulated data set with both relationality and correlation and compared their results to the true schematic structure of the simulated data, which is known by design. The results confirm that this switch from "Relational" to "Correlational" Class Analysis (CCA) reliably increases the accuracy of the technique, so much so that when the two disagree, the odds that CCA's results are more accurate approach 17:1. I also documented that RCA introduces a strong distributional assumption that has not previously been noted in published work. When it is violated, the odds in favor of CCA's results further increase to 24:1.

Both RCA and CCA seek to measure the extent to which each pair of respondents employs the same cultural schema and use these measures to create groupings. But what if the sociocognitive processes giving rise to shared cultural schemas are actually nonlinear in character, contrary to the theoretical intuitions formalized in this article? Although the motivating examples behind relationality do not entail such a possibility, Goldberg nonetheless designed RCA to function even in the 
presence of complex nonlinearities in the data (Goldberg 2011:1433). Because the model elaborated here is only one theoretical possibility of how schematic cultural cognition may function, this kind of robustness may be a prudent goal. I thus conducted additional simulations to determine whether RCA is preferable over CCA when shared cultural schemas depart from the theorized linearity.

In the first three sets of simulations, which I term "sublinear," I investigated the possibility that some of the basic theorized relationships between schemasscaling, shift, or inversion-do not take place. In the second three sets, which I call "superlinear," I examined the possibility of schematic transformations that are more complex than those envisioned by the theory: high-degree polynomial transformations, multiway interactions, and independence between parts of the schema. The simulations show that CCA remains reliably more accurate than RCA under every scenario examined. While substantial departures from linearity cause CCA's average accuracy to decrease, RCA's average accuracy decreases to an even lower level. I found no evidence of settings where RCA is more accurate than CCA.

Finally, to explore the substantive consequences of switching from relationality to correlation, I revisited Goldberg's (2011) RCA analysis of the 1993 General Social Survey (GSS) musical taste data. The RCA analyses had detected three schematic classes: "Omnivore-Univore," "Contemporary-Traditional," and "Highbrow-Lowbrow." The CCA results confirm the first two of these schemas but contain two other schemas in place of RCA's third. I term these additional schemas "Anything (but) Country" and "Anything (but) Heavy Metal." These schemas more closely resemble the patterns of exclusionary omnivorousness documented by Bryson (1996) than the hierarchical Highbrow-Lowbrow logic described by Goldberg. Thus, the CCA analyses confirm broad outlines of RCA findings but also contain potentially important differences. I further compare these results via a widely known multiple-groups analysis technique from structural equation modeling (SEM), which can be used to provide a goodness-of-fit measure for inductively located heterogeneity (Boutyline and Vaisey 2017). The multiple-groups analysis indicated that CCA's classes have a far better fit to the GSS data than RCA's. I conclude by discussing how future work can further advance the methodological and theoretical project stemming from Goldberg's (2011) deeply innovative contribution.

\section{Shared Schemas}

RCA's conception of shared cultural schemas builds on relational theories of meaning in the tradition of de Saussure and Lévi-Strauss (e.g., Cerulo 1993; Emirbayer 1997). Such theories hold that the meaning of symbols in a cultural system rests not in the signs themselves but in the relationships among them. So, for example, the concept "hot" acquires its meaning from its opposition to "cold" rather than from any innate properties of the word itself (de Saussure [1916] 2013; Ritzer and Stepnisky 2013). The concepts that make up a single cultural domain then exist in semantic networks, in which each element gains its significance from its relationships to the other elements. For example, each of the concepts "mother," "father," "son," and "daughter" is defined in part through its distinction from the other three 
concepts and cannot be fully understood without understanding them all (LéviStrauss 1963). Such a configuration of concepts within a single cultural domain can be thought of as a shared cultural schema-an abstract cognitive structure that individuals acquire through experience or acculturation.

As I noted above, Goldberg (2011) does not formally define what it means for a set of survey respondents to share such a cultural schema. ${ }^{2}$ He instead illustrated this relationship by way of a motivating example and accompanying diagram, which I recreated in Figure 1. Describing this figure, Goldberg stated that A and B have "identical" patterns of musical tastes, C's pattern is "almost a mirror image" of A's and B's, and D's is "different but not antithetical." He thus concluded that "respondents A, B, and C exhibit the same logic of musical taste construction...as they all exhibit the same structure of relevance and opposition" (Goldberg 2011:1405), whereas respondent $\mathrm{D}$ does not.

To arrive at a formal definition of schematic similarity, I expand Goldberg's discussion of this introductory example by writing out the implied algebraic operations. Respondent A likes pop, blues, and rock, strongly likes classical and opera, and is indifferent toward bluegrass and country: $A=[4,4,4,5,5,3,3]$. Respondent $\mathrm{B}$, on the other hand, dislikes pop, blues, and rock, is indifferent toward classical and opera, and strongly dislikes bluegrass and country: $B=[2,2,2,3,3,1,1]$. Except for an overall downward shift in the appraisal of all the genres, this pattern of tastes is identical to that of the first respondent: $B=A-2$.

In contrast to $A$ and $B$, respondent $C$ is indifferent toward pop, blues, and rock, strongly dislikes classical and opera, and strongly likes bluegrass and country: $C=[3,3,3,1,1,5,5]$. These tastes again follow the same relative pattern as $\mathrm{A}$ and $\mathrm{B}$ except all tastes are vertically shifted, inverted, and amplified: $C=2 *(-1) * A+$ 11 , or equivalently, $C=2 *(-1) * B+7$. Finally, respondent $\mathrm{D}$ strongly dislikes pop and rock, strongly likes blues, likes classical, opera, and bluegrass, and dislikes country: $D=[1,5,1,4,4,4,2]$. Unlike A, B, and C, this respondent construes an opposition between bluegrass and country but not between bluegrass and opera. No series of inversions, multiplications, or shifts of this pattern can transform it into the one exhibited by $\mathrm{A}, \mathrm{B}$, and $\mathrm{C}$. We thus conclude that whereas respondents $\mathrm{A}, \mathrm{B}$, and $\mathrm{C}$ follow the same schema, respondent $\mathrm{D}$ does not.

From this example, we can surmise that two respondents follow exactly the same schema if (i) their attitudes are identical, (ii) their attitudes are exact inverses of each other's, (iii) the attitudes of either respondent are uniformly more extreme than those of the other, (iv) the attitudes of either respondent are uniformly more positive than those of the other, or (v) any combination of (ii), (iii), and (iv). These conditions specify the mathematical operations of identity $(Y=X)$, inversion $(Y=-X)$, scaling $(Y=k X)$, and vertical shift $(Y=X+b)$ (see Figure 2$)$. They can thus be captured by a single algebraic statement: two respondents (X and $Y$ ) follow exactly the same schema if and only if there exists a linear transformation that can produce one vector of responses from the other one or, more formally, if there exist such constants $b$ and $k \neq 0$ that $Y=k X+b$. It is therefore intuitively clear that any measure of schematic similarity between two respondents should obtain its maximum value when such $k$ and $b$ exist and should otherwise capture 


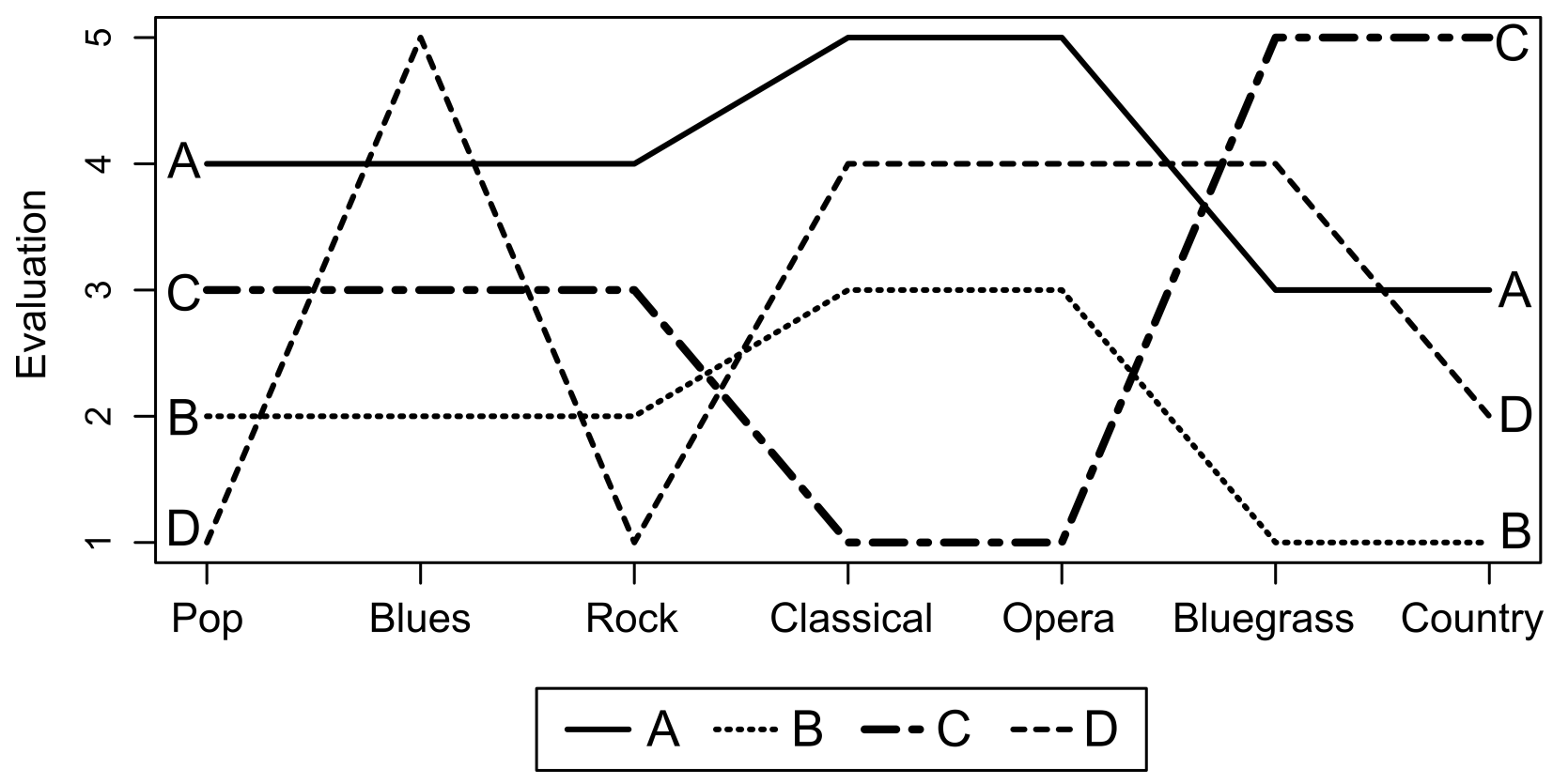

Figure 1: Musical tastes of four respondents, with evaluations ranging from 1 (strongly dislike) to 5 (strongly like) for each genre. Respondents A, B, and C follow the same taste schema, whereas D does not. This figure recreates the contents of Goldberg's Figure 1A (2011:1405).

the degree to which one pattern can be approximated by linear transformations of the other.

This model of schematic cognition thus formalizes the implied operations that give Goldberg's illustrative example of schematic similarity its intuitive plausibility. Because of the simplicity of these linear operations, it is easy to imagine the social processes that could bring about such schematic transformations. For example, a highly opinionated person may follow the same pattern of genre tastes as a less opinionated one but turn all "likes" into "strong likes" and all "dislikes" into "strong dislikes," thus yielding situation (iii); a music lover may begin with a pattern of musical tastes common to his or her social group but shift all of them upward to yield situation (iv); a rebellious teenager could begin with his or her parents' musical tastes and invert all of them to yield situation (ii); and so on.

Because many aspects of physical reality are linear (or approximately linear) in character, similar algebraic transformations can also be found in other cognitive domains. For example, in visual object perception, individuals are capable of recognizing the same stimulus placed in different positions and orientations (e.g., an apple that is rotated onto its side and moved farther away, which makes it appear smaller). This process has been theorized to involve the perceiver effectively carrying out matrix-algebraic transformations of the perceived object so as to match it with representations of objects stored in memory (Ullman 1989; Hummel 2013). The simplicity of the three basic cognitive transformations also fits with the "cognitive miser" model of mental processing, which proposes that human minds seek to minimize cognitive effort (e.g., Taylor 1981; Kool et al. 2010). The theorized 


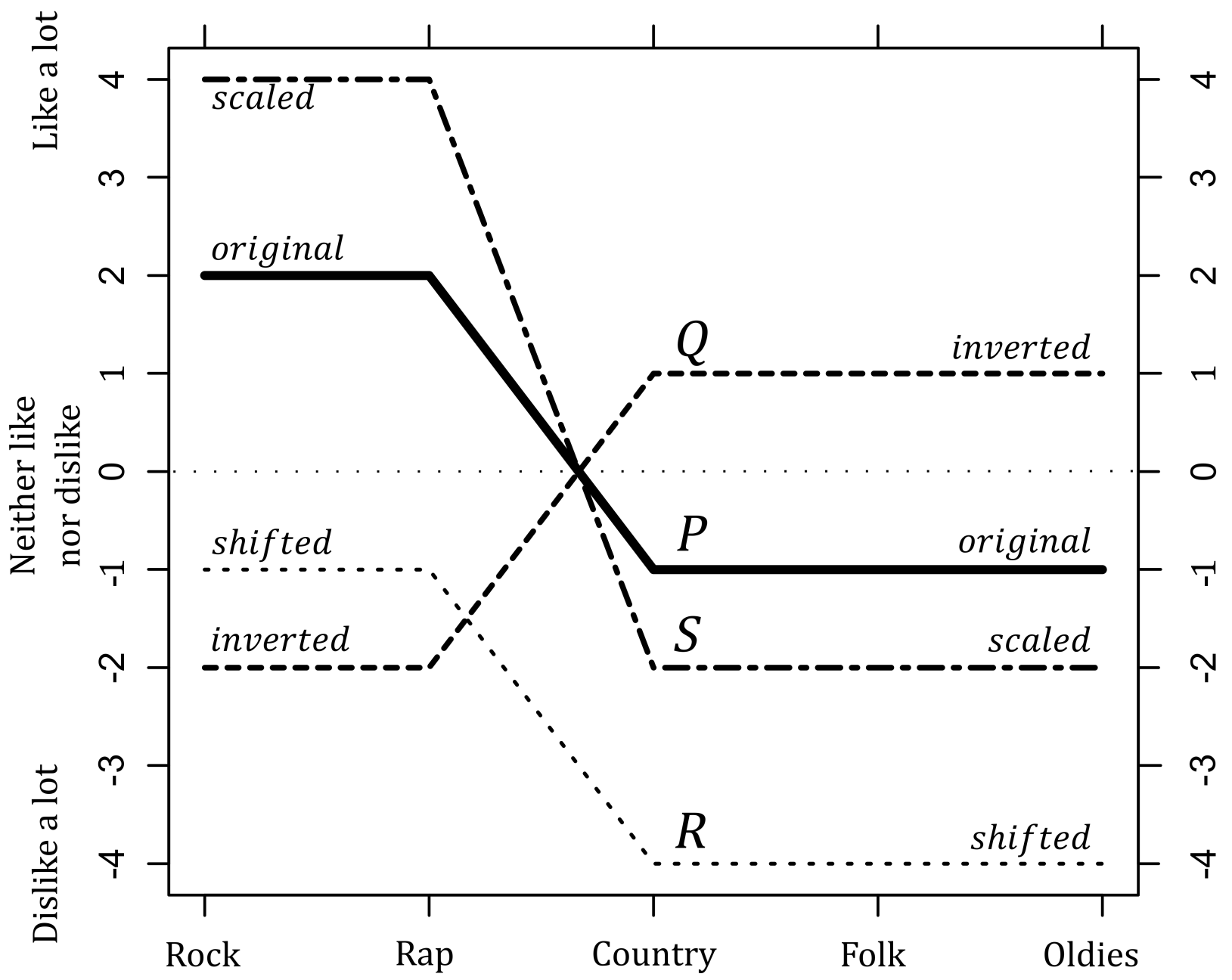

Figure 2: Original schema with the three basic linear schematic transformations.

transformations of taste schemas captured in this model thus appear both socially and cognitively plausible.

\section{Relationality}

Following his motivating example, Goldberg (2011) offered relationality $R_{i j}$ as a measure of this schematic similarity. It is computed by first taking the row vector containing the attitudes belonging to a respondent and calculating the differences between each pair of that respondent's attitudes by subtracting them from one another. ${ }^{3}$ Each survey row $i$ is thus transformed into a square matrix $X_{i}$ of pairwise arithmetic differences between variables in that row. Then, to calculate the relationality between a pair of respondents $i$ and $j$, the absolute values of their respective difference matrices $X_{i}$ and $X_{j}$ are elementwise subtracted from each 
other. Each element of the resulting matrix $D_{i j}$ is given a sign based on whether the corresponding entries of $X_{i}$ and $X_{j}$ were in the same or opposite directions. Finally, the elements of matrix $D_{i j}$ are summed together to yield the relationality $R_{i j}$, which is rescaled to range from 1 to -1 .

The distinction between positive and negative relationalities is not useful for RCA because either extreme indicates that respondents $i$ and $j$ follow the same schema. Thus, following a bias-reduction step that I examine later, RCA uses only the absolute values of relationality $\left|R_{i j}\right|$, which range from 1 (same schema) to 0 (unrelated schemas). RCA interprets the absolute relationalities as an adjacency matrix for a weighted network with respondents as nodes and their pairwise absolute relationalities as ties. Finally, it uses a modularity-maximization algorithm (Newman 2006) to partition this network into groups of respondents who have relatively high absolute relationalities $\left|R_{i j}\right|$.

Goldberg's (2011) argument that relationality measures schematic similarity is discursive rather than formal. Drawing on the structuralist idea that symbols acquire their meaning from their distinctions from other symbols, he argued that "[c]omparing how two individuals organize meaning therefore requires examining the associations between their attitudes. This calls for a method that looks at the extent of dissimilarity between the pairwise differences between their individual opinions" (p. 1403). Therefore, he constructs relationality around an across-respondent comparison of within-respondent arithmetic differences in genre ratings, apparently reasoning that because the shared schemas specify the relative value individuals assign to musical genres, they would be captured by such subtractions. But that reasoning is based on a conflation of distinction (semantic difference) and subtraction (arithmetic difference) - two concepts that are related in some contexts but substantially different in others. To illustrate the problem with this conflation, consider an analogy to melody perception, which is another cultural domain in which individual elements are defined relative to one another. Instead of the question, "Are two individuals who report different musical tastes nonetheless following the same schema in different ways?" the question here becomes, "Are two individuals who play different notes nonetheless performing the same melody in different keys?"

As is commonly known, most people perceive only relative rather than absolute pitch: that is, rather than discerning the absolute sound frequency of a musical note, they perceive its frequency relative to other notes played. Thus, if one were to change the key a melody is performed in - that is, to multiply all the tone frequencies in the melody by the same constant-most listeners would perceive the result to be identifiably the same melody (Radocy and Boyle 2012). ${ }^{4}$ Algebraically, if an audible frequency $k$ is taken to be the note $A$ in an equal-tempered musical scale, then the following two notes in the scale $(B b$ and $B)$ would have frequencies $k \times \sqrt[12]{2}$ and $k \times \sqrt[12]{2} \times \sqrt[12]{2}=k \times \sqrt[6]{2}$, respectively. For any melody, changing the value of $k>0$ would alter the scale's key but keep the melody recognizably the same (because, e.g., for any $k \neq 0, B / A=\sqrt[6]{2}$ ). Therefore, within the constraints of human hearing, two tone sequences $\left(x_{1}, y_{1}, z_{1}\right)$ and $\left(x_{2}, y_{2}, z_{2}\right)$ would be recognizable as the same melody as long as $y_{1} / x_{1}=y_{2} / x_{2}$ and $z_{1} / y_{1}=z_{2} / y_{2}$. An algorithm that determines whether two individuals are performing the same melody in different 
keys would thus need to effectively carry out an across-performer comparison of within-performer ratios (geometric differences) between the tone frequencies. If it were to instead compare the melodies by subtracting the tones from one another, as RCA does with musical tastes, it would generally get the comparison wrong.

The goal of this analogy is not to argue that subtraction-based relationality is necessarily the wrong measure for schematic similarity. Rather, it is to point out that distinctions between elements in a relative system may not be subtractive in character. We thus cannot conclude that relationality $\left|R_{i j}\right|$ is a valid measure of schematic similarity simply because it compares within-respondent arithmetic differences in tastes. Its ability to correctly detect shared schemas must instead be tested directly by applying it to sets of response patterns that either do or do not follow the same schema, and examining whether it can correctly determine which are which. ${ }^{5}$ Goldberg's introductory example discussed above can be used as such a test case.

Recall that in the introductory example, respondents A, B, and C followed exactly the same schematic pattern, whereas $\mathrm{D}$ followed a different one (Goldberg 2011:1405). The values $\left|R_{i j}\right|$ obtains in the introductory example should thus clearly identify that A, B, and C share a schema but D does not. However, relationality's difficulties with this task are evident in Figure 1B of the original article (Goldberg 2011:1405). I depict the relevant parts of this diagram ${ }^{6}$ in Figure 3, in which diagonally shaded bars represent $\left|R_{i j}\right|$. Relationality achieves its maximum value for the respondent pair $\mathrm{A}$ and $\mathrm{B}(\mathrm{AB}=1.00)$, thus clearly indicating that the two follow the same schema. Conversely, the relationality between the pair A and D is approximately 0.2 , which is appropriately low because $\mathrm{A}$ and $\mathrm{D}$ follow different schemas. Because $C$ follows the same schema as $A$ and $B$, the absolute relationalities $A C$ and $B C$ should optimally be equal to the same value as $A B(1.00)$. Unfortunately, this is not the case: both $\mathrm{AC}$ and $\mathrm{BC}$ have absolute relationalities of approximately 0.3 , which is far closer to the relationality of the unrelated pair $\mathrm{AD}(0.2)$. Thus, relationality appears to grossly understate the schematic similarity between respondent $\mathrm{C}$ and respondents $\mathrm{A}$ and $\mathrm{B}$.

To determine whether this inaccuracy affects the solution yielded by RCA, I created a data set consisting entirely of rows A, B, C, and D, each repeated 200 times for a total of 800 rows. I then analyzed it with the RCA software provided by Goldberg (Goldberg and Zhai 2013). To produce the correct solution, RCA would have to partition this population in two classes, the first containing all the copies of rows A, B, and C (600 rows total) and the second containing all copies of D (200 rows). However, RCA instead produced an erroneous solution consisting of three distinct classes, with the copies of $C$ incorrectly assigned to their own class separate from the copies of $A$ and $B^{7}$. Since Goldberg used this example to introduce relationality as a tool for detecting shared schemas, RCA's inability to do so here is troubling. ${ }^{8}$

\section{Correlation}

This shortcoming means that there is merit in trying out a different measure of schematic similarity. Recall that two respondents, $X$ and $Y$, follow exactly the same 


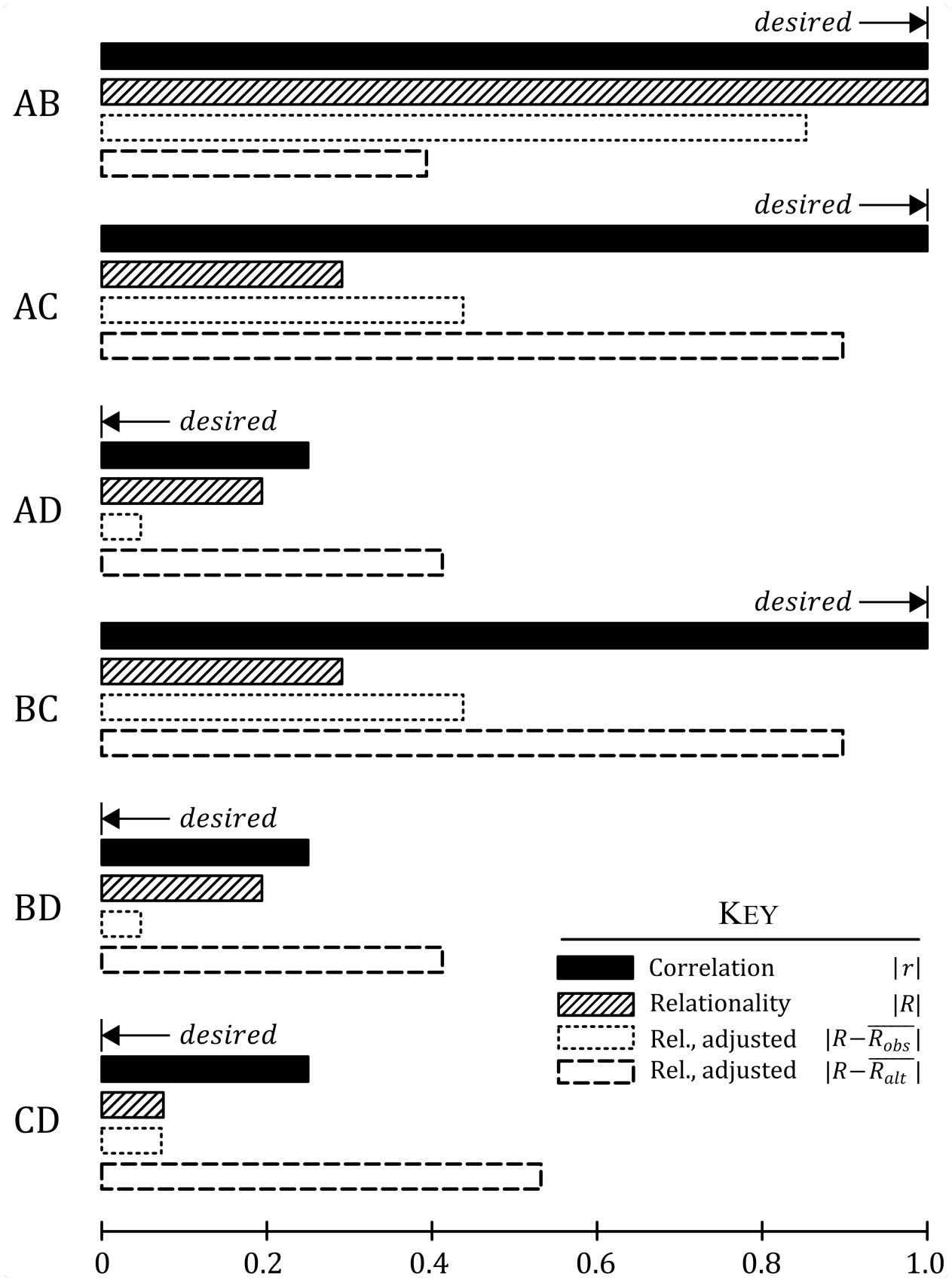

Figure 3: Absolute values of pairwise correlations (solid black) and relationalities (diagonal hatch) among the four response patterns from Figure 1 . The goal is to correctly determine that $\mathrm{A}, \mathrm{B}$, and $\mathrm{C}$ belong to one schematic class but $\mathrm{D}$ does not. The desired arrows indicate the values of each comparison that would best lend themselves to this correct answer. The remaining bars depict $\left|R_{i j}\right|$ after bias adjustment if each pattern occurs once (dotted) and if patterns A and B occur three additional times (dashed). 
schema if there exist such constants $k \neq 0$ and $b$ such that $Y=k X+b$. Thus, provided that $X$ and $Y$ have a finite nonzero variance, it can be easily shown that the absolute Pearson's correlation $|r|$ between $\mathrm{X}$ and $\mathrm{Y}$ equals 1 if and only if they follow exactly the same schema. As the two responses become more and more linearly independent of one another-that is, as the best possible linear transformation of $X$ leaves an ever-larger percentage of Y's variance unexplained-the value of $|r|$ decreases monotonically toward 0 . Finally, $|r|$ will be equal to 0 if and only if $k=0$ gives the best linear approximation of $\mathrm{Y}$, or in other words, if the best linear approximation of $Y$ ignores the contents of $X$ altogether. This is why Pearson's correlation is often interpreted as the "measure of the degree of linear relationship between two variables" (Stockburger 2011; see Rodgers and Nicewander 1988 for a detailed treatment). Thus, Pearson's correlation appears to be a perfect candidate for this task.

The solid bars in Figure 3 demonstrate the results obtained by applying Pearson's correlation to the same problem. The absolute correlations $\mathrm{AB}, \mathrm{AC}$, and $\mathrm{BC}$ all equal 1 , whereas $\mathrm{AD}, \mathrm{BD}$, and $\mathrm{CD}$ equal 0.25 . The absolute correlations between responses that follow the same schema are thus at their theoretical maximum, whereas the ones between members of different schematic classes are closer to their minimum. Thus, correlation appears to produce a far clearer depiction of the schematic relationships between these respondents than relationality. To examine whether this improvement results in a correct partition into classes, I adapted Goldberg's technique to use row correlations in place of relationalities. I term the resulting algorithm CCA (see Appendix A of the online supplement for details). And indeed, when I applied CCA to the same 800-row data set, it correctly assigned all the rows into the two schematic classes present in the data. Thus, whereas RCA failed to correctly recover the schematic classes in Goldberg's example, CCA produced a perfect answer.

\section{Simulation}

The above analysis suggests that CCA is a more accurate tool than RCA for detecting groups of respondents whose tastes follow the same cultural schema. However, one may rightly object that a single example does not provide a sufficient basis for drawing such a broad conclusion. To rule out the possibility that CCA's apparently superior performance is due to features specific to Goldberg's introductory example, I turned to simulation to carry out a more thorough analysis of the accuracy of both methods. In each of the 10,000 simulation runs reported in this section, I created a data set in which simulated respondents arranged their tastes according to one of a set of randomly generated shared cultural schemas by a process consistent with the theoretical model described above (in later sections, I examine simulations that violate the assumptions of this model). Because the schematic class membership of each respondent is known by design, these simulations enabled me to test how accurately CCA and RCA assign respondents to schematic classes under the conditions described by the theory.

The formal definition of shared schemas can serve as the basis for such simulations. Because two response vectors follow exactly the same schema if and only if 
they are linear transformations of one another, the schema that specifies relationships between $N$ tastes can itself be specified with a vector $\rho=\left[\rho_{1}, \rho_{2}, \ldots, \rho_{N}\right] .{ }^{9}$ Such a schema-specifying vector (hereafter "schema") can be randomly generated by drawing a vector of integers from an appropriate probability distribution. In turn, each response vector $X$ that exactly follows this schema can be generated by randomly drawing a pair of linear transformation constants $k$ and $b$, which can invert, rescale, or shift the pattern in ways consistent with the theory discussed earlier. Because real survey respondents do not perfectly reproduce cultural schemas, a simulated response must also include substantial stochastic deviations from the schema. These can be introduced via an independent error vector $\epsilon$ of the same length as the original pattern so that $X=k \rho+b+\epsilon$. This is the basic formula behind the first two sets of simulations I examined. To ensure that the simulations covered a wide range of potential cases, the simulation procedure contains three randomization steps. The first step of each run randomizes the broad characteristics of the simulated data set, such as the ranges and variances that will be used to generate the values of $\rho, k, b$, and $\epsilon$ as well as the number of distinct taste schemas behind the responses. The second step generates these schemas using the variance parameters produced in the first step. The final step generates a random number of respondents following each of these schemas by applying random linear transformation and adding random noise, both generated using the ranges and variances set in the first step. I repeated the entire procedure 5,000 times ${ }^{10}$ and created simulated data sets that widely differ in the ranges of simulated variables, variance of individual responses, signal-to-noise ratio, and many other parameters. A more detailed description of the simulation procedure can be found in Appendix $\mathrm{B}$ of the online supplement. An $R$ implementation of all the simulations described in this article is also available online.

Figure 4 illustrates a single simulation run. The randomly determined parameters from the first step of the run set the schema variance to 0.51 , the number of schemas to 3, and the maximum error variance, shift, and scaling to 1.02, 1, and 2 , respectively. Thus, to make the schemas $A, B$, and $C$, the simulation drew vectors from the normal distribution with $\mu=0$ and $\sigma^{2}=0.51$ and rounded them to the nearest integer. The resulting schemas are depicted with solid black lines with one per plot. For each schema, the simulation created a set of followers by randomly picking a value of shift $b$ from $\{-1,1\}$, scaling and inversion factor $k$ from $\{-2,-1,1,2\}$, and a noise vector $\epsilon$ drawn from the normal distribution with a variance of no more than 1.02. A small sample of such respondents is depicted in dashed lines behind the appropriate schema.

\section{Measuring Accuracy}

Each of the 5,000 simulated data sets generated by this procedure consists of randomly generated respondents who arranged their tastes according to one of a set of randomly generated taste schemas in a fashion consistent with the formal model I developed here. Thus, as in Goldberg's (2011) introductory example, the true schematic class membership for each simulated respondent is known by design. The simulation's goal is to assess the accuracy with which the group assignments 

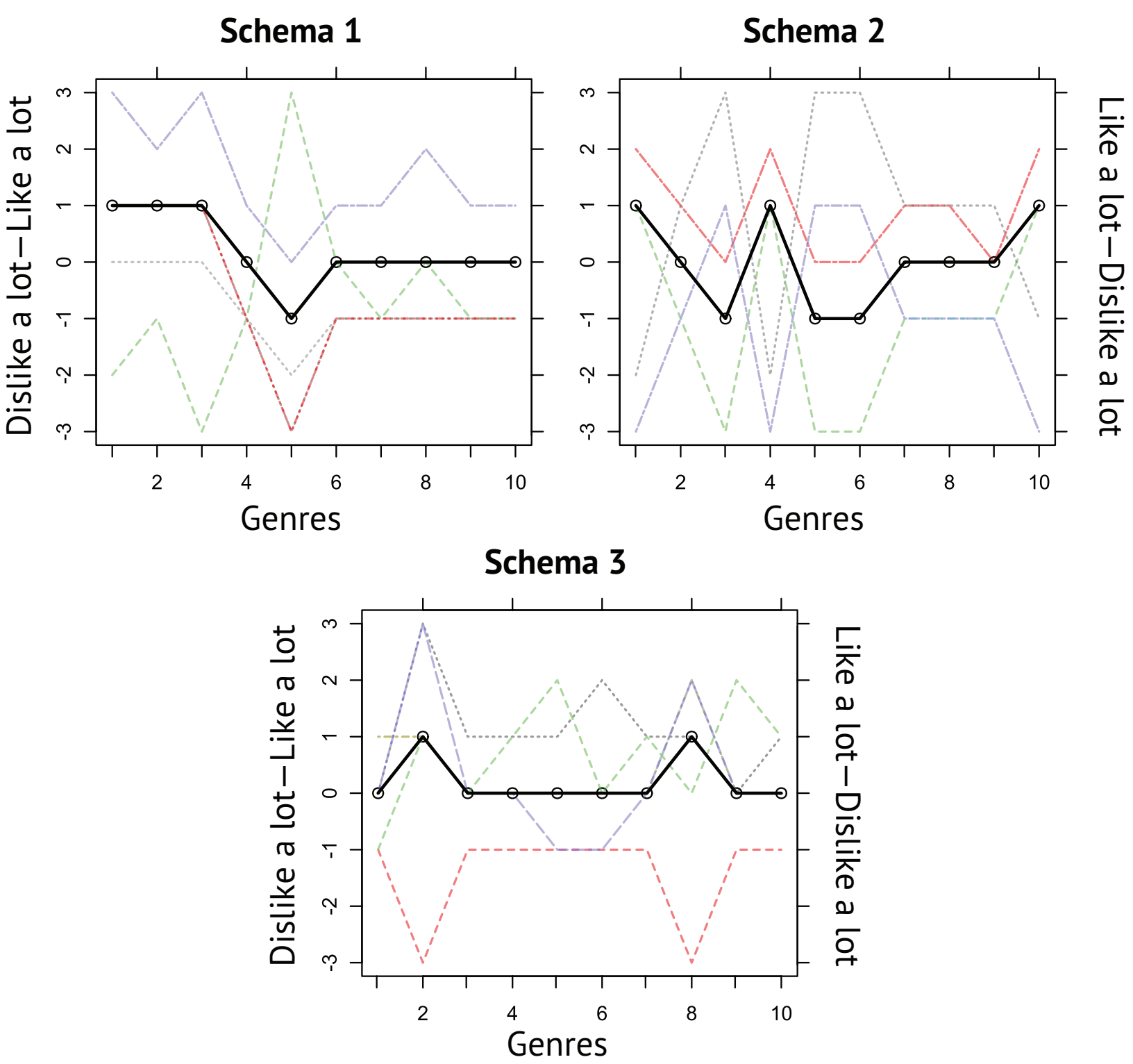

Figure 4: Three simulated schemas (solid black) for one simulation run with a small sample of responses derived from each schema depicted behind each one in the same plot (dashed). In this run, the pattern variance was 0.51 , and the maximum noise variance for individual responses was 1.05 . This moderately high ratio of noise-to-schema variance creates a relatively difficult classification task.

made by the two algorithms correspond to this known membership. If two respondents were generated from the same schema, they should be assigned to the same group; if they were created from different schemas, they should belong to different groups.

For each run, I measured this classification accuracy of each algorithm with normalized mutual information (NMI): 


$$
N M I(\Omega, C)=\frac{2 * I(\Omega ; C)}{H(\Omega)+H(C)},
$$

where $C$ is the vector of true class memberships for every respondent, $\Omega$ contains the corresponding class assignments made by the algorithm, $I$ is mutual information, and $H$ is Shannon entropy. NMI is an established criterion for measuring the accuracy of network-partitioning algorithms (e.g., Danon et al. 2005; Lancichinetti, Fortunato, and Kertész 2009). It ranges from 1 when the estimate $\Omega$ perfectly recreates the true membership structure $C$ to 0 when $\Omega$ is independent with respect to these true schematic classes (Manning, Raghavan, and Schütze 2008). ${ }^{11}$

\section{Simulation Results}

The results of these 5,000 tests are presented in Table 1 under the heading "Simulation 1." The median accuracy of CCA (0.97) is higher than that of RCA ${ }^{12}(0.74)$, a difference that is highly significant statistically (Wilcoxon $W=8,585,271, p<0.0001$ ). The interquartile range (IQR) of CCA's accuracy extends from 0.69 to 0.97 , whereas RCA's extends from 0.54 to 0.88 . Thus, whereas CCA's 75th percentile is just shy of perfect accuracy, RCA's 75th percentile barely surpasses CCA's median. The substantive significance of these differences is clearer when the CCA accuracies $(Y)$ are plotted against the RCA accuracies $(X)$ in Figure 5. Although the accuracies are strongly associated $\left(R^{2}=0.79\right)$, CCA is more accurate than RCA in the vast majority of cases (88.1 percent). In contrast, RCA is more accurate than CCA in only 5.2 percent of the cases. Thus, when RCA and CCA disagree, which they do in 93.3 percent of the cases, the odds that CCA's result is more accurate than RCA's equal $17: 1$.

To determine if the results point to any classes of data in which RCA would nonetheless be preferable to CCA, I disaggregated them by schema variance and noise variance, which are the parameters most responsible for the difficulty of the classification task. Lower schema variances or higher noise variances result in more challenging signal-to-noise ratios, which should make the performance of both algorithms poorer. The loess curves demonstrating this effect are presented in Figure 6.

On both plots, the accuracies of the two algorithms overwhelmingly rise and fall together, thus suggesting that the algorithms find the same cases challenging and the same cases easy. In spite of this similarity, CCA's accuracy remains reliably above RCA's throughout the full ranges of both variances, with a median gap of roughly 0.10 in favor of CCA. This accuracy gap remains remarkably stable throughout most of the two ranges. For example, in simulations within the top 5 percent of noise variance, the median accuracies equal 0.61 for CCA and 0.51 for RCA. Within the bottom 5 percent of noise variance, they equal 1.00 and 0.91 , respectively. CCA thus retains the same advantage over RCA under both the least and the most challenging noise conditions examined.

The only instance in which the two accuracy curves substantially deviate from each other occurs when the schema variance is low. In such situations, the pairwise correlations between responses tend toward zero, which is a well-known bias, 
Table 1: Comparison of RCA and CCA accuracy in 10,000 simulation runs.

\begin{tabular}{|c|c|c|c|c|}
\hline \multirow[b]{2}{*}{ Measure } & \multicolumn{2}{|c|}{$\begin{array}{l}\text { Simulation } 1 \\
(5,000 \text { runs) }\end{array}$} & \multicolumn{2}{|c|}{$\begin{array}{l}\text { Simulation } 2 \\
\text { (5,000 runs) }\end{array}$} \\
\hline & $\begin{array}{l}\text { Relationality } \\
\text { (RCA) }\end{array}$ & $\begin{array}{l}\text { Correlation } \\
\text { (CCA) }\end{array}$ & $\begin{array}{l}\text { Relationality } \\
\text { (RCA) }\end{array}$ & $\begin{array}{l}\text { Correlation } \\
\text { (CCA) }\end{array}$ \\
\hline Overall accuracy (median NMI) & 0.74 & 0.87 & 0.67 & 0.87 \\
\hline $\begin{array}{l}\text { Accuracy, interquartile range } \\
(25 \% \text { to } 75 \%)\end{array}$ & $(0.54,0.88)$ & $(0.69,0.97)$ & $(0.46,0.84)$ & $(0.69,0.97)$ \\
\hline $\begin{array}{l}\text { Runs with near-perfect } \\
\text { accuracy (NMI > 0.95) }\end{array}$ & $13.2 \%$ & $30.5 \%$ & $9.2 \%$ & $30.3 \%$ \\
\hline $\begin{array}{l}\text { Runs with near-complete } \\
\text { inaccuracy (NMI > 0.05) }\end{array}$ & $2.8 \%$ & $0.1 \%$ & $3.1 \%$ & $0.1 \%$ \\
\hline $\begin{array}{l}\text { Runs with higher accuracy } \\
\text { than other method }\end{array}$ & $5.2 \%$ & $88.1 \%$ & $3.8 \%$ & $91.6 \%$ \\
\hline $\begin{array}{l}\text { Odds of higher CCA accuracy } \\
\text { when results differ }\end{array}$ & \multicolumn{2}{|c|}{ 16.9:1 } & \multicolumn{2}{|c|}{ 23.8:1 } \\
\hline
\end{tabular}

Notes: Results are from simulations with randomly varying schema variances, noise amounts, and ranges of linear transformations. In simulation 2 , inversion odds also randomly varied.

while their relationalities approach one. Therefore, as Goldberg pointed out, the relationality between low-variance respondents is systematically higher than the correlation. When Goldberg briefly considered using correlation in his online appendix A, he dismissed it on the basis of this difference. However, Goldberg incorrectly interpreted this difference to mean "relationality does a better job at examining relationships between respondents whose responses have relatively low variance" (Goldberg 2011:Appendix A). The fact that relationality produces higher values than correlation does not imply that it produces more accurate values. And indeed, as can be seen on the left side of Figure 6A, the opposite appears to be true. When schema variance is in the lowest 5 percent of its simulated range, CCA's median accuracy decreases to 0.31 . However, RCA's accuracy experiences a disproportionally large drop and decreases to 0.07 . This low accuracy indicates that RCA results for low-variance schemas contain almost no information about the true membership structure of the data. It further suggests that relationality may exhibit an upward bias for low-variance observations that is substantially more damaging to its performance than correlation's downward bias. ${ }^{13}$

\section{Distributional Assumptions}

Relationality also introduces a strong distributional assumption that may further degrade its accuracy when violated. Whereas a correlation of zero always indicates an absence of a linear relationship, the equivalent "null value" of relationality differs from data set to data set and is generally skewed above zero (Goldberg 2011:Appendix A). RCA attempts to compensate for this bias by recentering the matrix of relationalities by its mean. This bias-adjustment procedure crucially rests on the assumption that the true mean relationality between all the rows in the data is zero or, equivalently, that the relationality values are distributed symmetrically 


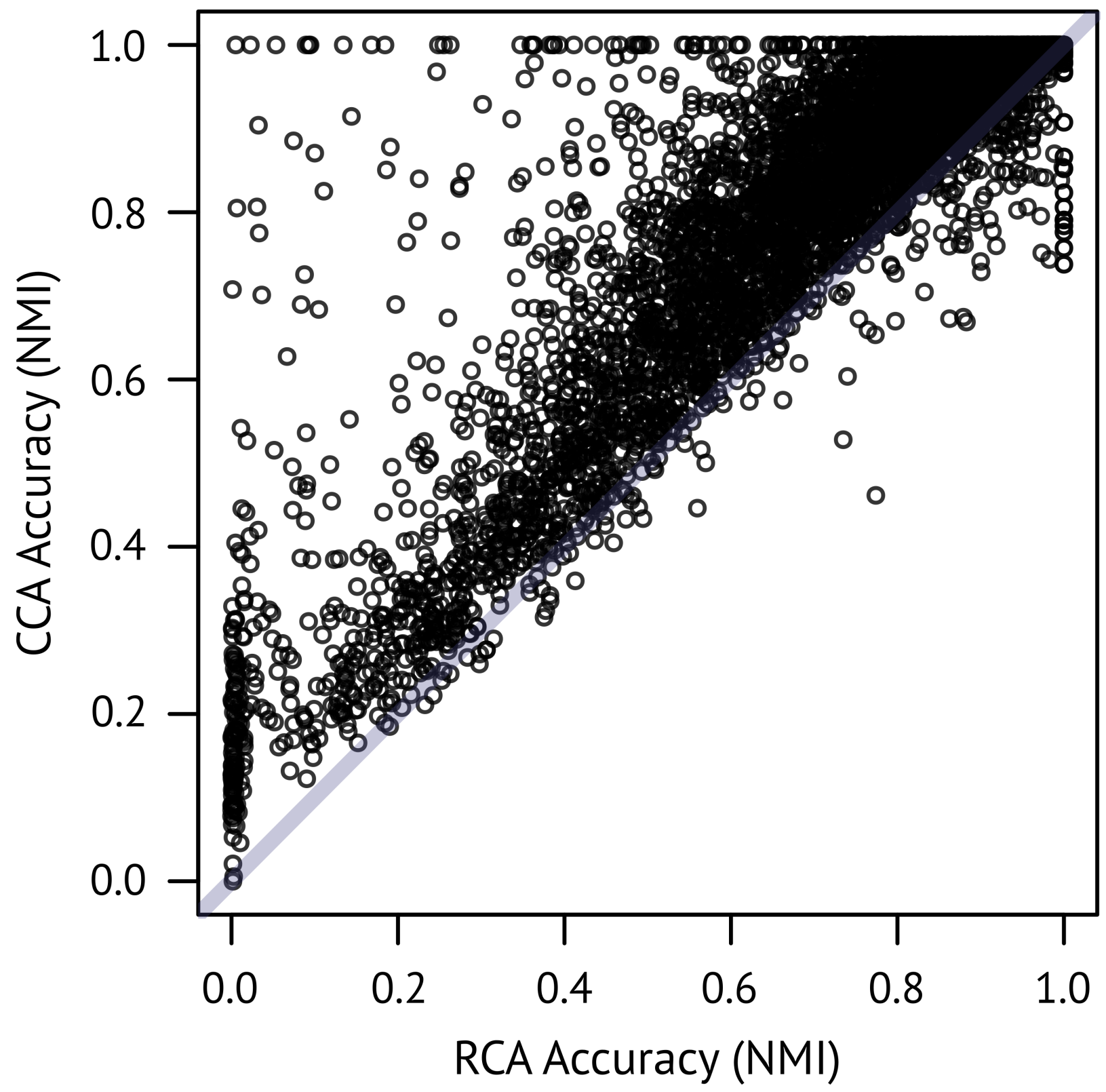

Figure 5: CCA accuracy compared with RCA accuracy for 5,000 simulation runs. Each point is a single run. Runs in which CCA was more accurate are above the $Y=X$ diagonal (in gray), and those in which RCA was more accurate are below. Note the absence of points in the bottom-right corner.

around their null value. This would normally be the case only if the proportion of respondents following a schema without inverting it equaled the proportion following its inverse ${ }^{14}$ — a quantity I call "inversion probability." For example, when this probability is 50 percent, the number of highbrow respondents following the schema "like classical, like opera, dislike rock, dislike country" would equal the number of lowbrow respondents following its inverse, "dislike classical, dislike 

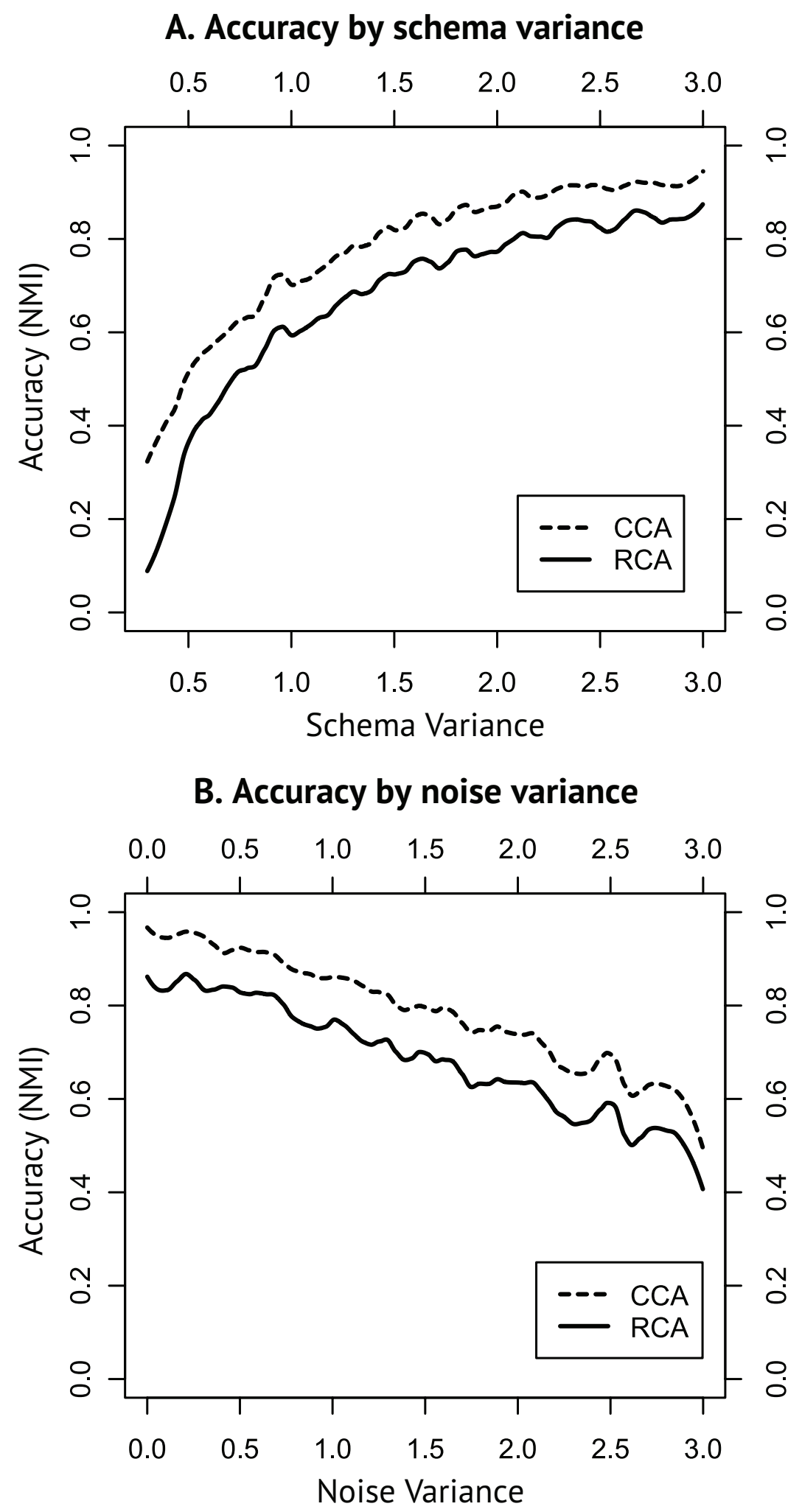

Figure 6: Loess curves comparing RCA and CCA accuracy based on 5,000 simulation runs. 
opera, like rock, like country." However, because in reality the number of highbrow respondents can differ greatly from the number of lowbrow respondents, there is no reason to expect that the inversion probability generally equals 50 percent. This suggests that RCA's symmetry assumption may be frequently violated by empirical data.

To illustrate the magnitude of potential error that occurs when this assumption is violated, I return to the introductory example. In Figures 1 and 3, the mean relationality between the four respondents equals $\overline{R_{o b s}}=(A B+A C+A D+B C+$ $B D+C D) / 6 \approx 0.15$. Subtracting this value from the other relationalities yields the adjusted relationalities $\left|R-\overline{R_{o b s}}\right|$, which are depicted in dotted lines in Figure 3. In this adjusted result, the related pairs $\mathrm{AC}$ and $\mathrm{BC}$ are assigned higher relationalities than before, whereas the unrelated pairs $\mathrm{AD}, \mathrm{BD}$, and $\mathrm{CD}$ are decreased. Aside from the relationality of the related pair $\mathrm{AB}$, which is also decreased by the bias adjustment, the bias-adjusted scores $\left|R-\overline{R_{o b s}}\right|$ appear to be a clear improvement over the unadjusted values of $|R|$.

However, the bias-adjustment procedure can easily have the opposite effect. Consider an alternative data set that is just like the one in the introductory example but with the addition of three more copies each of the patterns A and B. In this alternative data set, the mean relationality would then be $\overline{R_{\text {alt }}} \approx 0.6$. The relationality matrix that is adjusted for this new mean, $\left|R-\overline{R_{a l t}}\right|$, is depicted in dashed lines in Figure 3. This adjustment correctly brings the relationalities between $\mathrm{AC}$ and $\mathrm{BC}$ closer to 1 . However, it also causes a large decrease in the relationalities between the related pair A and B, which are identical except for a vertical shift. It further substantially increases the estimated relationalities between unrelated pairs AD, $\mathrm{BD}$, and $\mathrm{CD}$. These completely unrelated patterns are now erroneously assigned a higher relationality than the closely related pair $\mathrm{AB}$. As this example illustrates, RCA's bias-adjustment procedure crucially depends on the relative number of times each pattern appears in the observed data set-a quantity that is fundamentally arbitrary.

All the simulations presented above have granted RCA's assumption of symmetric distribution of relationalities by keeping the inversion probability fixed at 50 percent. To examine the effects of relaxing this assumption, I created a second simulation in which the inversion probability is drawn instead from a uniform distribution over its full range and varies between each of the 5,000 simulation runs. ${ }^{15}$ The results of this simulation are reported in Table 1 under the heading "Simulation 2." As expected, both the median (0.87) and the IQR (0.69 to 0.97) of CCA accuracies remain unchanged from the first simulation. On the other hand, the median accuracy of RCA drops to 0.67 , which is significantly lower than its prior median of 0.74 (Wilcoxon $W=13,802,245, p<0.0001$ ) and below CCA's 25th percentile of accuracy. CCA is now more than three times as likely as RCA to produce a nearly perfect answer (NMI > 0.95), whereas RCA is more than 20 times as likely to produce an almost completely incorrect one (NMI $<0.05)$. Thus, RCA's accuracy appears to suffer a further significant drop when its assumption of symmetrical distribution is violated, as may generally happen in empirical applications. CCA remains unaffected by this change. ${ }^{16}$ 
The results of both simulations thus reinforce my earlier suppositions. Although the accuracies of RCA and CCA were highly correlated, they nonetheless differed in almost 95 percent of the 10,000 combined simulation runs. In simulation 1, which obeyed RCA's symmetry assumption, the odds that CCA's result would be more accurate equaled 17:1. In simulation 2, in which this assumption was relaxed, these odds further rose to 24:1. CCA was more accurate over the full range of schema and noise variances examined. The only major deviation from this otherwise stable gap in accuracies occurred in simulations with low schema variance. In those simulations, RCA performance suffered a significant additional drop relative to CCA's. Overall, the simulations provided no evidence of any use cases in which RCA would be preferable to CCA.

\section{Deviations from the Model}

The 10,000 simulations I described above demonstrate that CCA yields substantially more accurate results than RCA when the shared cultural schemas result in linear dependencies between rows in the survey data set. Such linear dependencies are consistent with the motivating examples of schematic similarity in Goldberg's (2011) work as well as the formal reasoning behind CCA I've laid out here. As I argued above, they are also socially and cognitively plausible. Nonetheless, schematic cultural cognition is little understood, and whether this model of schematic similarity as linearity is correct is not known. For this reason, the performance of both algorithms when this model is violated is also of interest. Another reason for this interest stems from the close conceptual relationship between correlation and linearity, which could potentially lead correlation-based CCA to perform disproportionally well in simulations that obey the linearity assumption. Since Goldberg may not have been aware that the examples he presented in his work were linear in character, he did not specifically design RCA to detect linear transformations. Simulations that violate the model would thus yield a more conservative test of CCA's accuracy and could potentially point to use cases in which RCA has an advantage over CCA. For these reasons, I now turn to simulations in which respondents' patterns are produced through processes that differ from the linear transformations theorized here.

There are two fundamentally distinct ways that schematic transformations could deviate from the theorized model. First, schematic transformations could in reality be based on only a subset of the possible linear operations (shift, scaling, and inversion). Although such cases would technically remain linear, the two algorithms may perform differently on these "edge cases" of linearity than they do with all three operations present. ${ }^{17}$ Secondly, schematic transformations could be based on operations which are nonlinear in character, such as in the presence of multiway interactions between variables. I will use the shorthand terms "sublinear" and "superlinear" to refer to these two broad kinds of deviations from the model. In this section, I examine six distinct sets of simulations to investigate both of these possibilities. 


\section{Sublinear Transformations}

Recall that musical-taste schemas are theorized to consist of relative evaluations of different musical genres. Because these evaluations are relative rather than absolute, individuals can use one schema to produce a variety of contrasting taste patterns. Specifically, they can invert the schema, turning likes into dislikes; they can scale it, making all the appraisals uniformly more or less extreme; and they can vertically shift it, making all the appraisals more positive or negative. ${ }^{18}$ There is, to my knowledge, no alternative theory that clearly articulates a different set of cognitive processes that may underlie this kind of schematic cognition. However, there are plausible scenarios in which each of the three theorized transformations would not exist. And, given the noisiness of much survey data, a method that searches for rare or nonexistent schematic transformations could generate a substantial amount of false positives and thus yield inaccurate results. In the absence of empirical work documenting that the three theorized processes do indeed take place, it appears useful to investigate whether the methods are robust to the potential absence of these transformations. In this section, I use three sets of simulations to do so.

I begin by briefly sketching some potential reasons to question the prevalence of the basic linear operations of scaling, inversion, or shift. My goal here is not to argue that any of these operations are likely to be absent but rather to point out that such an absence is plausible enough to warrant further attention. To do so, I return to Figure 2, which illustrates each of the three basic linear transformations in isolation.

The patterns in this Figure are all derived from one shared schema (labeled "original"). Respondent $P$ follows this schema without any transformations and thus likes both rock and rap $(+2)$ and mildly dislikes country, folk, and oldies $(-1)$. Respondent $Q$ uses the same schema as $P$ but inverts it to create the opposite pattern of tastes $(Q=-1 * P)$. The cognitive demands of such an inversion are minimal because it simply requires taking a pattern of tastes and replacing the likes with dislikes. However, whether two social actors with very different taste patterns like highbrow and lowbrow would actually employ exactly the same cultural schema is uncertain. Because cultural taste is intertwined with social position, individuals with dramatic cultural differences are also likely to live in different kinds of places, consume media from different sources, and have network ties to distinct kinds of alters (Bourdieu 1987; Fararo and Skvoretz 1987; McPherson, Smith-Lovin, and Cook 2001; McPherson 2004;). This social distance suggests that rather than inverting the same taste schema, two groups with contrasting tastes may employ separate cultural schemas that maintain only an indirect, imprecise, symbolic, or accidental opposition to one another.

It is also possible to raise questions regarding the empirical prevalence of vertical shifts like the one employed by respondent $R(=P-3)$. Whereas the original schema consisted of a pattern of contrasting positive and negative appraisals, $R$ 's pattern contained only genres she dislikes a little (rock and rap $=-1$ ) and those she dislikes a great deal (country, folk, and oldies $=-4$ ). Such a transformation could occur if respondent $R$ had internalized the same set of distinctions as $P$ but simply did not enjoy listening to music, thus giving lower appraisal to all the genres. However, internalizing cultural distinctions takes effort and time (e.g., Bourdieu 
1987). It is thus reasonable to wonder if respondents who dislike music would generally put in the effort to internalize the same complex set of genre contrasts as music lovers, who presumably have a far greater intrinsic motivation to expose themselves to music.

The case of respondents with multiplicatively scaled response patterns like $S(=P * 2)$ could raise similar questions. Unlike $P$, who perceived relatively mild distinctions between musical styles, $S$ 's tastes feature dramatic contrasts between very strong positive assessments of some genres and very negative assessments of others. Again, this invites the same concern regarding generally unequal levels of motivation.

These questions about the empirical prevalence of the three kinds of linear transformations will need to be settled by future empirical work. In the meantime, they merit investigating how CCA and RCA would perform in the scenario that each of these transformations does not take place. Among the second set of 5,000 simulations I reported earlier in the article, 1,185 featured no vertical shift $(b \in\{0\})$, and 1,700 featured no scaling $(k \in\{1\})$. To examine the performance of CCA and RCA in the absence of these transformations, I reanalyzed these cases in isolation. I also performed 1,000 further simulations to examine the performance of the two algorithms in the absence of schematic inversion $(\zeta=0)$.

I report the accuracy of CCA and RCA in the three sublinear scenarios in Table 2. Among the "no shift," "no scaling," and "no inversion" simulations, CCA had median accuracies of $0.84,0.82$, and 0.87 , respectively. These were slightly lower on the average than its previous accuracy of 0.87 (Table 1 , simulation 2), but the difference is minor. With RCA, the "no shift," "no scaling," and "no inversion" simulations yielded accuracies of $0.68,0.66$, and 0.41 , respectively. Whereas the first two of these scores were close to RCA's previous accuracy of 0.67 , its score in the "no inversion" scenario was almost 40 percent lower. RCA was thus capable of retaining its previous accuracy in the "no shift" and "no scaling" scenarios but not in the "no inversion" scenario. This large drop in accuracy may again originate with RCA's strong assumption of symmetric distribution as discussed above.

Comparing the CCA and RCA accuracies on each individual run showed that CCA was more accurate in 88 percent, 88.5 percent, and 99 percent of the scenarios that omitted shift, scaling, and inversion, respectively. Thus, CCA remains the preferred choice of method if there is doubt regarding the empirical prevalence of some of the theorized linear transformations and especially so if these doubts concern schematic inversion. In Appendix $C$ of the online supplement, I also show how Pearson's formula can be altered to produce modified coefficients that specifically reflect each of these three sublinear scenarios. The three modified coefficients treat inversion, shift, or scaling as schematic difference rather than schematic similarity. Although CCA performed well in each of these settings, it may be possible to use such specially adjusted correlation coefficients to increase its accuracy even further while retaining the method's speed and simplicity. 
Table 2: Comparison of RCA and CCA accuracy in six sets of simulations with departures from theorized model.

\begin{tabular}{|c|c|c|c|c|}
\hline Simulation & $\begin{array}{c}\text { RCA accuracy } \\
\text { (median and IQR) }\end{array}$ & $\begin{array}{c}\text { CCA accuracy } \\
\text { (median and IQR) }\end{array}$ & $\begin{array}{l}\text { Methods } \\
\text { equally accurate } \\
\text { (\% of runs) }\end{array}$ & $\begin{array}{c}\text { CCA } \\
\text { more accurate } \\
\text { (\% of runs) }\end{array}$ \\
\hline \multicolumn{5}{|l|}{ Sublinear: } \\
\hline No shift & $\begin{array}{c}0.68 \\
(0.46,0.86)\end{array}$ & $\begin{array}{c}0.84 \\
(0.65,0.96)\end{array}$ & $5.4 \%$ & $88.0 \%$ \\
\hline No scaling & $\begin{array}{c}0.66 \\
(0.44,0.84)\end{array}$ & $\begin{array}{c}0.82 \\
(0.61,0.95)\end{array}$ & $5.1 \%$ & $88.5 \%$ \\
\hline No inversion & $\begin{array}{c}0.41 \\
(0.21,0.57)\end{array}$ & $\begin{array}{c}0.87 \\
(0.69,0.97)\end{array}$ & $0.3 \%$ & $98.7 \%$ \\
\hline \multicolumn{5}{|l|}{ Superlinear: } \\
\hline Polynomial functional form & $\begin{array}{c}0.27 \\
(0.16,0.38)\end{array}$ & $\begin{array}{c}0.42 \\
(0.31,0.57)\end{array}$ & $0.1 \%$ & $94.2 \%$ \\
\hline Independent subschemas & $\begin{array}{c}0.49 \\
(0.31,0.69)\end{array}$ & $\begin{array}{c}0.69 \\
(0.49,0.85)\end{array}$ & $1.1 \%$ & $76.1 \%$ \\
\hline Multiway interactions & $\begin{array}{c}0.23 \\
(0.12,0.42)\end{array}$ & $\begin{array}{c}0.52 \\
(0.26,0.79)\end{array}$ & $0.1 \%$ & $95.8 \%$ \\
\hline
\end{tabular}

\section{Superlinear Transformations}

It is also conceivable that shared cultural schemas result in schematic transformations that are more complex than scaling, shift, and inversion. The theory of schematic similarity elucidated here does not predict the existence of such transformations. However, Goldberg intended relationality to function even in the presence of such nonlinear relationships between variables (e.g., Goldberg 2011:1433) and argued that the complex way that relationality is computed makes it "more sensitive to interdependencies" between variables than correlation (Goldberg 2011:Appendix A). In this section, I use further simulations to investigate whether relationality's complexity indeed makes it more accurate than correlation in the presence of such superlinear transformations.

There are at least three broadly distinct kinds of conceivable superlinear transformations: polynomial (rather than linear) functions, independence between parts of the taste schema, and multiway interactions between tastes. All three of these scenarios contradict the theory of schematic similarity developed here. All three also violate different assumptions made by Pearson's correlation, thus creating potential difficulties for CCA with respect to RCA. To be clear, these three superlinear scenarios are not backed by well-articulated theoretical models of shared cultural schemas like the one developed in this article. These simulations are thus general tests of robustness to potential unforeseen deviations from the theorized model rather than examinations of concrete alternative use cases for either method. ${ }^{19}$

Functional form. I begin by using polynomials to examine deviations from the model that can come from more complex functional forms. High-degree polynomials are straightforward to generate randomly and can be used to approximate 
a wide variety of other continuous functions, with the maximum degree of the polynomial providing the limit on the number of inflection points and thus the complexity of the transformation. In contrast to the linear transformations, which always express clear, understandable processes of scaling, inversion, and shift, most of these random polynomial transformations have no clear theoretical justification. ${ }^{20}$ They are instead informative because of the diversity of transformations they produce. This scenario thus provides insight to the methods' performance under a broad range of unspecified deviations from the theorized model.

In these simulations, I replaced linear transformations with randomly generated polynomials of the form:

$$
X=g(\rho)=\sum_{i=0}^{10} a_{i} b_{i} \rho^{i}=a_{0} b_{0}+a_{1} b_{1} \rho+a_{2} b_{2} \rho^{2}+\cdots+a_{10} b_{10} \rho^{10},
$$

where $a_{i} \in\{-1,0,1\}$ and $b_{i}>0$. The $a_{i}$ coefficients determine whether a polynomial term is present as well as whether it is inverted, and the $b_{i}$ coefficients determine its relative weight. I adapted the three-step randomization process described earlier to generate such polynomials. ${ }^{21}$

The results of 2,000 such simulations are reported in Table 2 with the label "Polynomial functional form." In these simulations, CCA obtained a median accuracy of 0.42 , which is roughly half its previous accuracy of 0.87 . RCA obtained a median accuracy of 0.27 , or roughly 40 percent of its previous accuracy of 0.67 . CCA yielded more accurate results in 94.2 percent of the runs. Thus, while polynomial transformations proved challenging for correlation, they appeared no less challenging for relationality.

In Figure 7, I disaggregated these results by the maximum degree of the random polynomial transformation in the simulation run, which tracks the complexity of the transformation. Some simulations were randomly assigned first-degree polynomial transformations, which are analogous to the linear transformations used before (albeit with different signal-to-noise ratios). The Figure shows that CCA and RCA had their highest accuracies with these first-degree transformations. The accuracy of both algorithms then dropped as the degree of the polynomial increased. However, CCA remained reliably more accurate than RCA throughout the entire range. These results thus yielded no evidence that RCA's computational complexity improves its ability to detect more complex functional forms of schematic similarity.

Independent Subschemas. All the reported simulations have thus far assumed that each cultural schema specifies the relationships among all the attitude objects. However, it is conceivable that some respondents may use distinct cultural "subschemas" to organize different subsets of their tastes. For example, they may use one subschema to specify the relationship between genres to which most Americans are frequently exposed on radio and television and a different subschema for genres encountered only in more specialized settings. Alternatively, they could use one subschema for genres they were exposed to by their parents and another subschema for genres they learned about through friends. Such respondents could then apply different transformations to different subsets of tastes (e.g., by inverting the subschema for popular genres without inverting the one for specialized genres). 


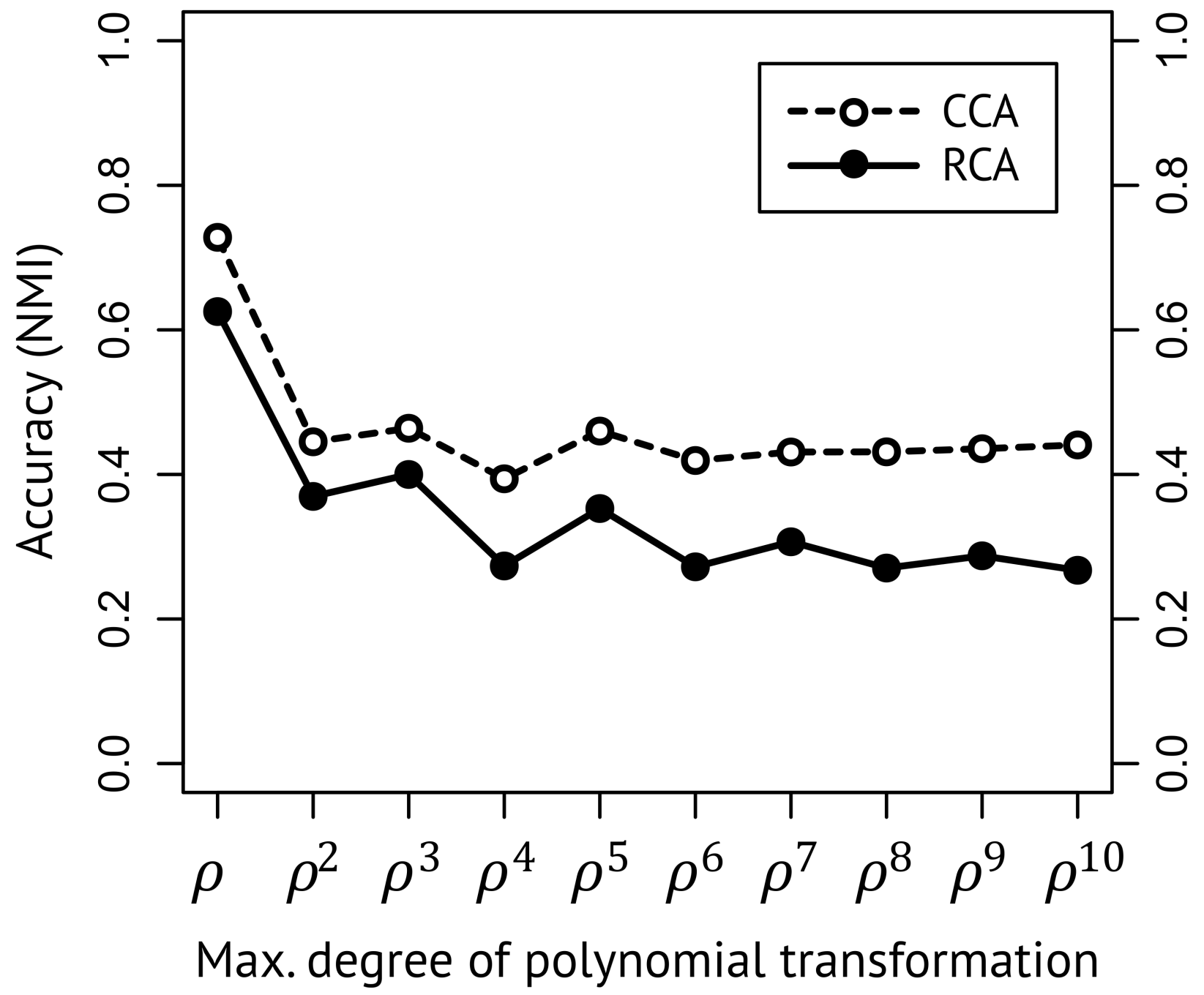

Figure 7: Accuracy for 3,000 simulations with polynomial functional form.

When different transformations are applied to different parts of a schema, they combine into overall schematic transformations that cannot be captured in terms of simultaneous deviations from each vector's single common mean. Whereas the correlation coefficient is computed from these deviations, relationality instead compares the deviations of variables from one another (Goldberg 2011:Appendix A), which could potentially give it an advantage under this kind of nonlinearity. Any such advantage, however, would be limited in its usefulness because both CCA and RCA assign each respondent to exactly one class that is assumed to correspond to exactly one schema and thus cannot possibly produce the correct solution in most situations with independent subschemas. ${ }^{22}$ Extending CCA and/or RCA to cover such cases would require substantial further theoretical and methodological development. 
In their present form, the methods can still be applied to an edge case of this scenario in which different subschemas cannot be arbitrarily recombined to produce an individual's pattern of tastes but rather always occur in combination with exactly the same subschemas. ${ }^{23}$ This is the scenario I simulate here. As this limitation greatly reduces its realism, this set of simulations is again presented only as a test of robustness rather than a theoretical alternative to the model of schematic similarity as linearity.

To examine this scenario, I simulated 2,000 data sets in which individual responses were derived from the schemas that contained up to four different subschemas inside them. ${ }^{24}$ I reported the results of these "independent subschemas" analyses in Table 2. In these simulations, the median accuracy of CCA was 0.68, and the median accuracy of RCA was 0.49 . Thus, for both CCA and RCA, this scenario proved substantially more challenging than the linear ones, in which their accuracies were 0.87 and 0.67 , respectively. Overall, CCA produced more accurate results in 76 percent of the runs.

In Figure 8, I disaggregated these results by the average count of independent subschemas within the schemas used in each simulation run, which tracks the extent to which a run deviates from the assumption of variance around a single common mean. The runs in which this count equaled 1 are analogous to the linear simulations analyzed above (albeit again with different signal-to-noise ratios). CCA's median accuracy in these single-subschema runs was 0.81 and RCA's was 0.55. The accuracy of both algorithms degraded as the number of subschemas grew, falling to 0.44 for CCA and 0.31 for RCA when this quantity reached the simulation maximum of 4. CCA again remained consistently more accurate than RCA throughout the full range of the simulation.

Multiway interactions. I now turn to potential departures from the model caused by interactions between variables in the taste schema. In the examples presented by Goldberg (2011) and the simulations reported above, each element of a respondent's vector $R$ was always produced from transformations of exactly one element of the schema $\rho$. For example, consider a respondent $R$ who begins with a taste schema $S=\left[S_{\text {rock }}=2, S_{\text {rap }}=x, S_{\text {classical }}=y, S_{\text {opera }}=z\right]$, then inverts it, scales it by 2 , and finally shifts it by +1 . This respondent is, in effect, applying the univariate function $R_{k}=f\left(S_{k}\right)=(-1) * 2 * S_{k}+1$ to each element of $S$. No matter what values $x, y$, and $z$ assume, $R^{\prime}$ s attitude toward rock would equal $f(2)=-3$.

In contrast, in the presence of interactions between attitudes, a given respondent's attitude regarding a single genre may be a function of two or more tastes in the schema-for example, $R_{\text {rock }}=g\left(S_{\text {rock }}, S_{\text {rap }}, S_{\text {classical }}\right)=\left(S_{\text {rock }}^{2} * S_{\text {rap }} * S_{\text {classical }}\right)^{\frac{1}{4}}=$ $\sqrt[4]{4 x y}$. The presence of such multiway interactions would entail complex and perhaps unintuitive relationships between cultural schemas and the responses they generate. ${ }^{25}$ In contrast to the linear transformations that are the focus of this article, it is difficult to imagine any plausible sociocognitive mechanism that would consistently yield such intricate transformations. However, in the interest of examining the robustness of both algorithms under a maximally broad range of unexpected departures from linearity, I used further simulations to study their performance in this setting. 


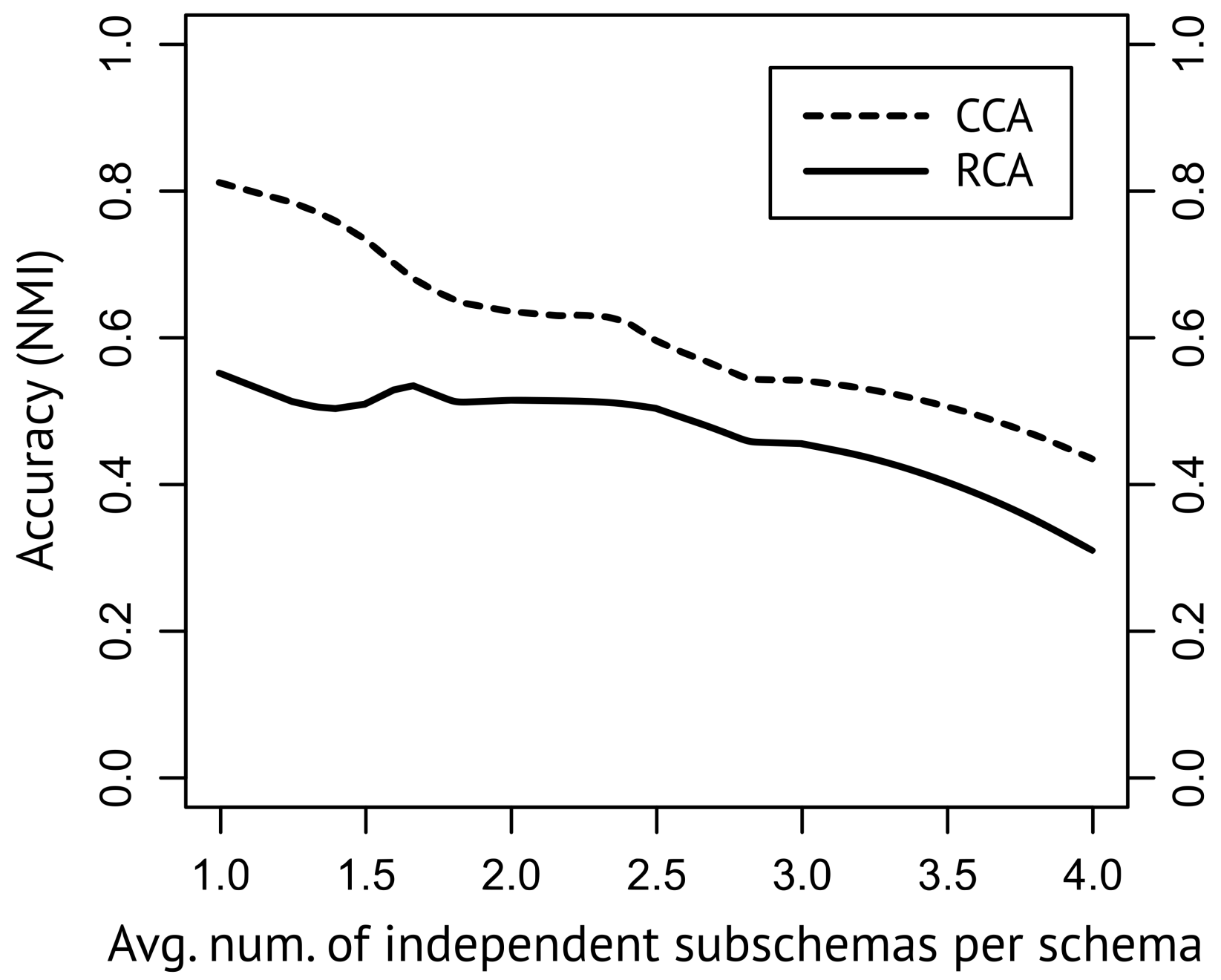

Figure 8: Accuracy for 2,000 simulations with independent subschemas.

In this procedure, each simulated respondent partitioned a shared schema into groups of tastes I term "interaction blocks," and then generated each of his or her genre tastes by calculating its randomly weighted geometric mean with other tastes in its block. ${ }^{26}$ Each respondent thus used a random transformation of the form similar to $g$ above-for example, $R_{k}=g(k, m, n, p)=\sqrt[6]{k^{3} m n p}$. Because each respondent was assigned idiosyncratic interaction blocks, each response vector consisted of multiplicative interactions between different sets of tastes. To create a range of complexities, the maximum number of tastes per interaction block differed at random from run to run.

I report the results of 2,000 runs of this simulation in Table 2 ("Multiway interactions"). The median accuracy was 0.52 for CCA and 0.23 for RCA, which indicates that correlation again yielded more accurate results than relationality. To examine how the complexity of interactions affected accuracy, I disaggregated the simulation 


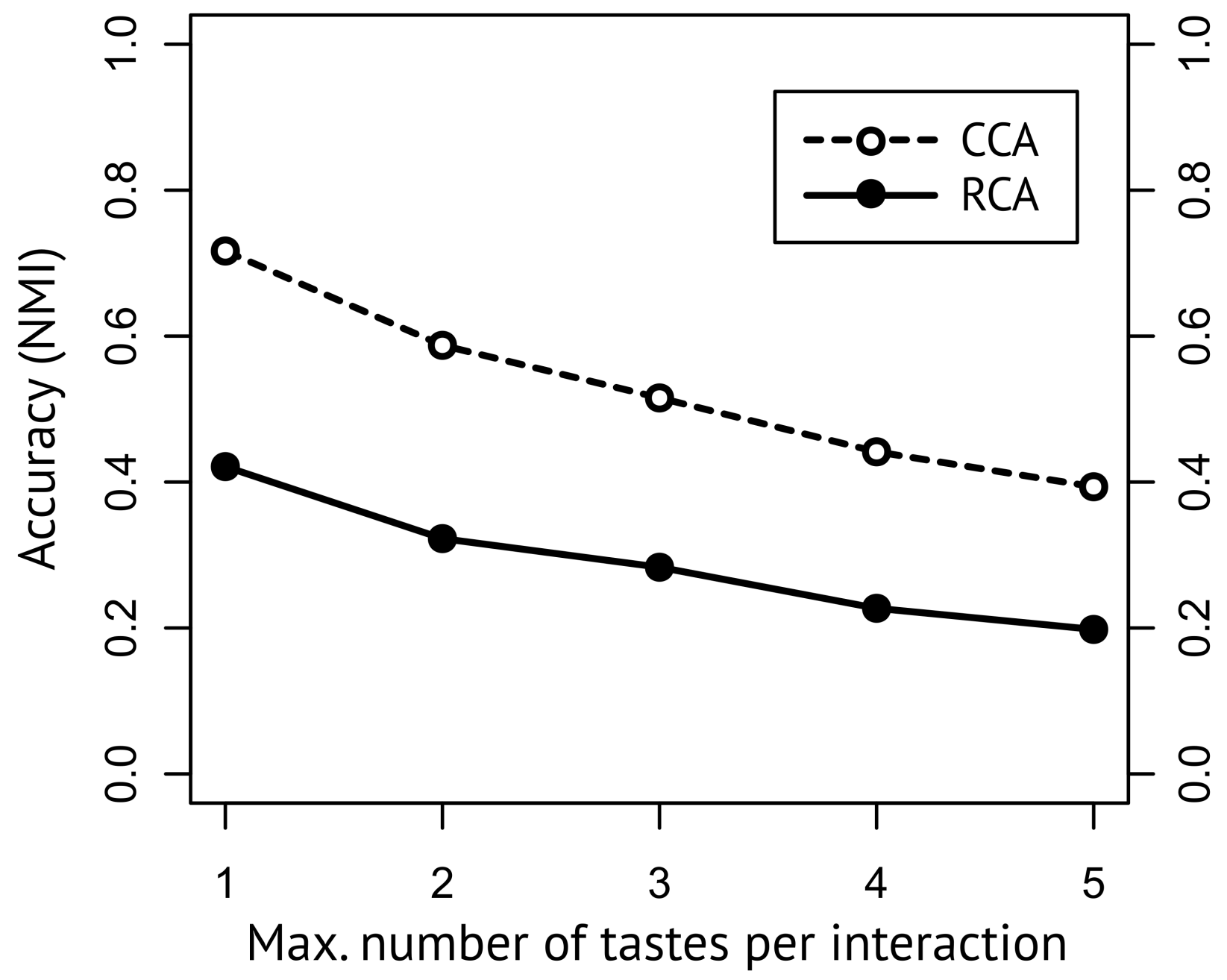

Figure 9: Accuracy for 2,000 simulations with multiway interactions.

runs by the maximum number of tastes per interaction block in the given run (see Figure 9). When this number equaled 1, the transformations added noise but did not actually feature any interactions between terms. As expected, this was the easiest scenario for both CCA and RCA, which achieved accuracies of 0.72 and 0.42 , respectively. These simulations became increasingly more challenging for both CCA and RCA as the number of tastes per interaction increases. By the time the interaction size reached its maximum of 5, both CCA and RCA saw their initial accuracies drop by roughly half to 0.39 and 0.20 , respectively. Throughout the 2,000 runs of this simulation, CCA yielded a more accurate result than RCA on 96 percent of the runs, which indicates that it is the preferable technique even in the presence of complex interactions.

The simulations I examined in this section compared three broad ways in which relationships between taste vectors can violate the assumptions of linearity. I examined situations in which linear transformation functions were replaced by polyno- 
mials, in which some parts of the taste schema were transformed independently from others, and in which transformations of the taste schema involved multiway interactions between separate tastes. Although Goldberg (2011) contended that relationality's computational complexity makes is better equipped than Pearson's correlation to handle complex relationships between variables, these simulations indicated that this is likely not the case. CCA yielded more accurate results than RCA in 94 percent of the simulations with random polynomial functional forms, 96 percent of the simulations with multiway interactions, and 76 percent of the simulations with independent subschemas (see Table 2). When I disaggregated each scenario by difficulty, I found that RCA's accuracy consistently trailed CCA's. The simulations thus yielded no evidence of cases-linear or nonlinear-in which RCA would be preferable to CCA. Moreover, it is interesting to note that in Figure 7, where the relationship between polynomial degree and classification accuracy was highly nonmonotonic, RCA's accuracy still repeatedly rose and fell in close synchrony with CCA's. The drops in accuracy again closely resembled each other in the presence of interactions (Figure 9). And, although the two methods responded differently to small numbers of independent subschemas, the drops in RCA's accuracy also closely paralleled CCA's through most of that simulation's range (right side of Figure 8). This remarkably persistent pattern suggests that minor differences aside, relationality may generally be detecting the same substantive relationships among respondents as correlation does, albeit at a consistently lower accuracy.

\section{Empirical Example: Musical Tastes}

To compare the results produced by the two methods in an empirical setting, I applied CCA to the 1993 GSS musical tastes module previously analyzed with RCA (Goldberg 2011). This data set contains 1,532 respondents' evaluations of 17 musical genres. Each respondent rated each genre using a five-point Likert scale that ranges from "like very much" to "dislike very much." For comparability, I followed exactly the same coding procedures as Goldberg (2011). Below, I contrast the substantive contents of the results. I then compare their fit to the data using a multiple-groups analysis technique from SEM.

The RCA analyses partitioned the survey population into three schematic classes, which Goldberg labeled "Omnivore-Univore," "Highbrow-Lowbrow," and "Contemporary-Traditional." For respondents in the Omnivore-Univore class, most genre tastes were positively correlated among each other. Goldberg interpreted this as evidence of a culturally omnivorous taste schema in which no genres are perceived as opposites, but rather a high appraisal of most genres is opposed to a low appraisal of most genres. In the Highbrow-Lowbrow class, tastes for "elitist" genres such as opera and classical music were positively correlated among each other but negatively correlated with most tastes for popular genres. Finally, Goldberg characterized the third schematic class as Contemporary-Traditional. Here, a cluster of positively correlated tastes for well-established musical genres including gospel, bluegrass, and country is negatively correlated to tastes for arguably more contemporary genres, including heavy metal, pop, and rap, as well as oldies and jazz. 


\section{A. Omnivore-Univore}

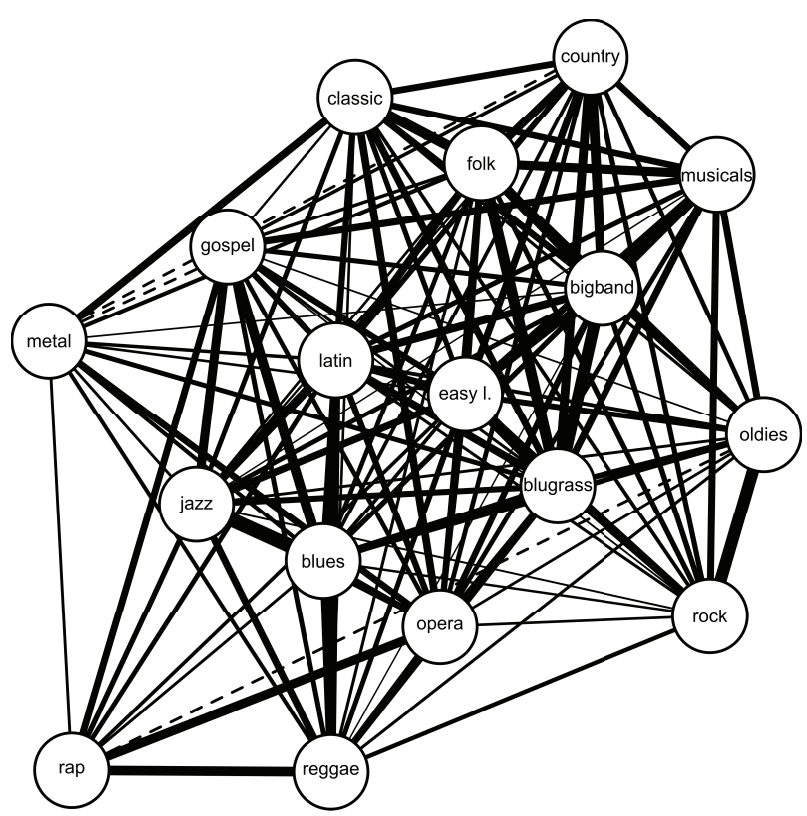

C. Anything (But) Heavy Metal

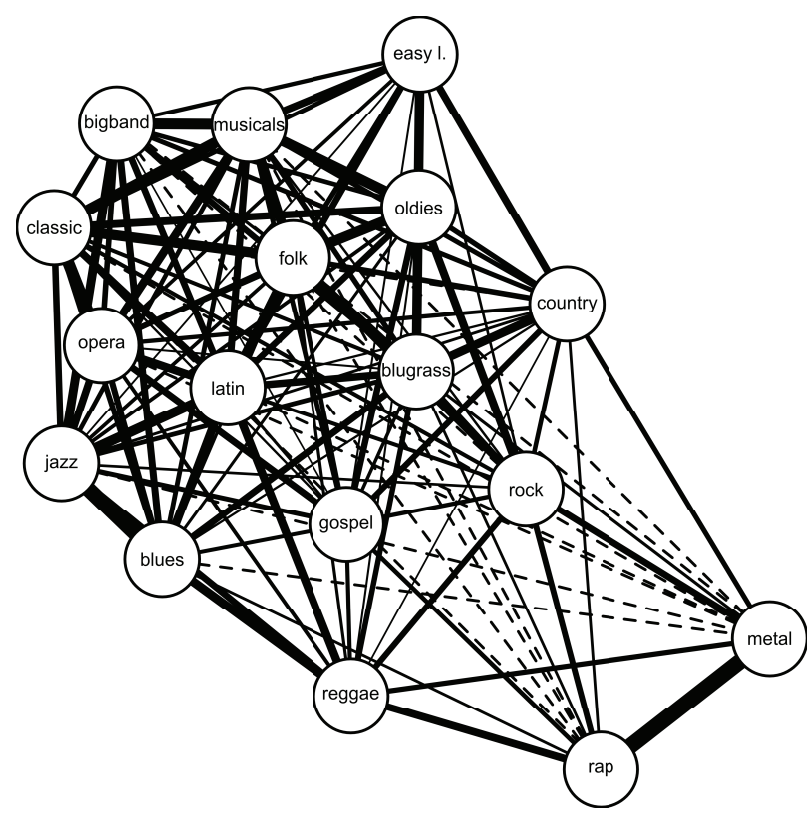

B. Contemporary-Traditional

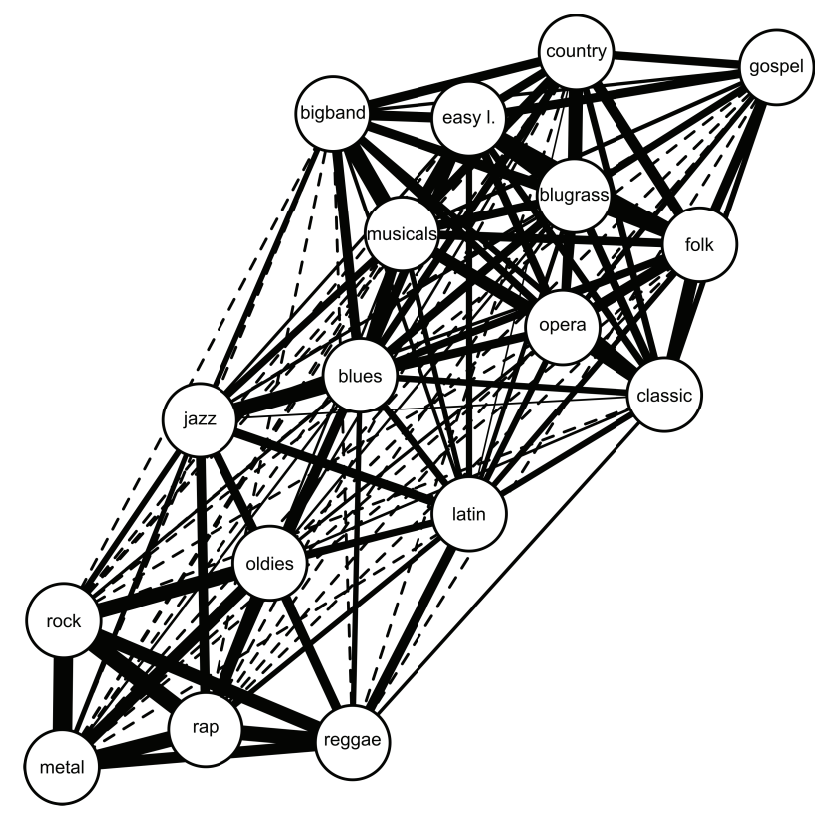

D. Anything (But) Country

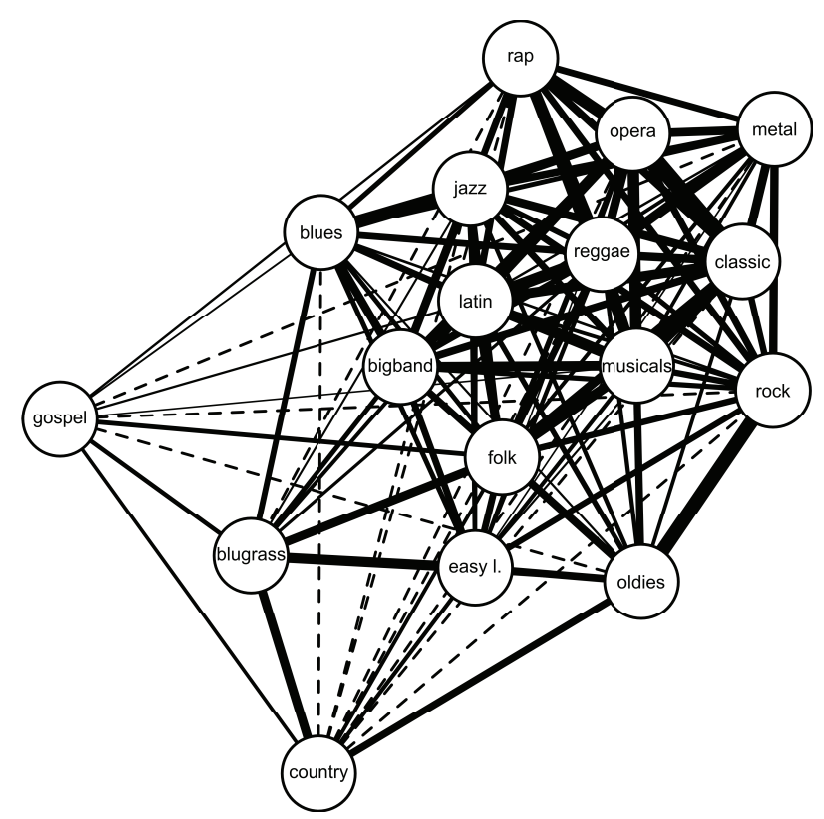

Figure 10: Networks illustrate the four schematic classes identified by CCA. Ties represent correlations between genre tastes, with stronger correlations indicated by thicker lines. Negative correlations are depicted with dashed lines. Weak correlations $(|r|<0.05)$ are not plotted. 
The persistence of the Highbrow-Lowbrow taste schema was perhaps Goldberg's most surprising finding because much contemporary work has argued that omnivorousness has replaced highbrow tastes as a marker of high status in the contemporary United States (Peterson and Kern 1996; Peterson 1997, 2005; see Goldberg 2011 for a more detailed discussion). The CCA analyses of these data, however, do not replicate this finding. Whereas RCA identified three classes in these data, CCA identified four, which are presented in Figure 10. The first two of these closely resemble those located by RCA. The first class features practically no negative correlations between the genres, which suggests that respondents in this class perceive little opposition between different musical styles. In this population, positive appraisal of any one genre generally "goes with" positive appraisal of any other genre, which suggests an undiscriminating logic of taste that ranges between near-uniformly positive appraisals of all genres on one extreme and a nearuniformly negative appraisal of all genres on the other. This is the same omnivorous logic as behind the Omnivore-Univore class identified by RCA.

The second class located by CCA appears to be defined by an opposition between rock, rap, and metal on one extreme and gospel, country, folk, and bluegrass on the other. This suggests a bifurcation of respondents into those who prefer newer musical genres and those who prefer more established ones, which closely resembles the logic of the Contemporary-Traditional class identified by RCA. The two methods, however, deviate in their classifications of blues and jazz, which RCA had categorized as "contemporary" rather than "traditional." In contrast, CCA analyses instead suggest that blues belongs to the traditional side of the divide, whereas jazz, along with Latin, straddles the two sides without clearly belonging to either. Because both blues and jazz were already well-established genres by the mid-twentieth century, the Contemporary-Traditional class identified by CCA fits better with the intuitive chronological understanding of those terms and may thus have greater face validity.

The biggest apparent difference between the CCA and RCA analyses concerns the remaining group of respondents. RCA had placed the remaining respondents into a single class that appeared to follow a traditional hierarchical logic, with highbrow genres such as opera and classical music on the one extreme and popular lowbrow genres on the other. In contrast, CCA separated the remaining population into two further schematic classes that appear to follow a different set of logics (see panels C and D of Figure 10). In both CCA-identified classes, the majority of genres were tied together in a dense cluster of positive correlations, thus suggesting that both are variants of an omnivorous taste schema. These results resemble previous findings that documented the existence of multiple distinct logics of omnivorousness (e.g., Tampubolon 2008).

However, the omnivorousness of respondents in these last two classes features clear exceptions. In the class depicted on the left, a higher appraisal of most genres is generally accompanied by a lower appraisal of heavy metal (and frequently also rap music.) The class on the right exhibits a nearly identical structure, except country and gospel music occupy the same position of exclusion as metal and rap did in the class on the left. These patterns closely echo the analyses of Bryson (1996), who famously showed that omnivores may retain a symbolic boundary against 
Table 3: Cross-tabulation of estimated schematic class memberships in 1993 GSS musical tastes data.

\begin{tabular}{|c|c|c|c|c|}
\hline & \multicolumn{4}{|c|}{ CCA Class } \\
\hline & $\begin{array}{l}\text { Omnivore- } \\
\text { Univore }\end{array}$ & $\begin{array}{c}\text { Contemporary- } \\
\text { Traditional }\end{array}$ & $\begin{array}{c}\text { Anything (But) } \\
\text { Country }\end{array}$ & $\begin{array}{c}\text { Anything (But) } \\
\text { Heavy Metal }\end{array}$ \\
\hline \multicolumn{5}{|l|}{ RCA Class } \\
\hline Omnivore-Univore (673) & 281 & 92 & 167 & 133 \\
\hline Contemporary-Traditional (394) & 60 & 241 & 35 & 58 \\
\hline Highbrow-Lowbrow (461) & 142 & 36 & 159 & 124 \\
\hline Total $(N=1,528)$ & 483 & 369 & 361 & 315 \\
\hline
\end{tabular}

Notes: Cross-tabulation of class memberships estimated by CCA (columns) and RCA (rows). RCA class sizes are indicated in parentheses. Four out of 1,532 respondents were omitted from this analysis because the variances of their responses equaled zero.

genres most closely associated with low education: heavy metal, rap, country, and gospel music. Thus, drawing on the title of Bryson's work, I term these latter two classes "Anything (but) Heavy Metal" and "Anything (but) Country."27

Table 3 cross-tabulates the group assignments made by the two algorithms. A plurality (though not a majority) of respondents that RCA grouped into its first two classes remained grouped together in the CCA results. The third class identified by RCA, however, does not have an analogous relationship to any one of CCA's classes. Such a divergence between CCA and RCA class assignments is consistent with the simulation analyses above, in which the results of the two methods were correlated but were rarely the same. While the simulations had demonstrated a reliable difference in accuracy between the two methods, these empirical analyses confirm that the two methods can point to substantively different conclusions.

\section{Multiple-Groups Analysis}

Both RCA and CCA are theoretically understood as methods for partitioning the population into groups of respondents who follow different cultural schemas. However, from a more practical perspective, their task is to detect unobserved heterogeneity in how attitudes or tastes correlate for different groups. This heterogeneity is exemplified by the hypothetical situation in which a pair of attitudes are correlated positively in one subgroup and negatively in another, which leads them to appear uncorrelated in the overall sample (e.g., Baldassarri and Goldberg 2014:56). RCA and CCA reveal these latent logics of taste organization by inductively partitioning the population into groups in which the same pairs of tastes correlate differently with one another. The sets of logics these methods locate are thus subsample correlation matrixes that were mixed together to yield the overall sample.

Their success in locating this kind of unobserved heterogeneity can be measured by how well these resulting subsample correlation matrixes describe the observed distribution of tastes in the population. A well-known multiple-groups testing technique from SEM can provide such a measure (Bollen 1989; review in MacCallum and Austin 2000). Multiple-groups analysis is widely used in psychology and public health to ascertain whether survey scales "have the same meaning across groups" 
(Gregorich 2006:S78), such as across age cohorts or education levels. Here, we are interested instead in ascertaining that the subgroups located by one of the methods show evidence of substantial differences in meaning.

To test the extent of this difference, I used SEM to evaluate whether using separate correlation matrixes for each subgroup meaningfully improves model fit over a single correlation matrix for the whole population, taking parsimony into account. The differences in Akaike information criterion (AIC) and Bayesian information criterion (BIC) between the single-matrix and multiple-matrix models can be used to quantify the significance of the heterogeneity detected by RCA or CCA. Roughly speaking, these indicators answer the question, "Are the groups located by these methods distinct enough in their logics of taste organization to justify partitioning the population into separate classes?" Because neither AIC nor $\mathrm{BIC}$ require the models to be nested, the same approach can also be used to compare the fit of RCA and CCA partitions to one another.

I used SEM to fit three models to the musical tastes data: a homogeneity model in which a single correlation matrix describes the whole population, a model with three correlation matrixes corresponding to RCA's partition of the population, and a model with four correlation matrixes corresponding to CCA's partition. The log likelihoods are $-35,376.85(\mathrm{df}=153)$ for the single-matrix model, $-34,054.2(\mathrm{df}=$ 459) for the RCA partition model, and $-33,381.32(\mathrm{df}=612)$ for the CCA partition model. Comparing their model fit via AIC and BIC, I found that both the RCA $(\triangle \mathrm{AIC}=-2,033.3 ; \Delta \mathrm{BIC}=-401.8)$ and $\mathrm{CCA}(\triangle \mathrm{AIC}=-3,073.07 ; \Delta \mathrm{BIC}=-625.81)$ partitions are strongly preferred to the single-matrix model. This is consistent with the existence of significant heterogeneity in the schemas that different subgroups use to organize their musical tastes. Both indicators also show that the CCA-based partition fits the data far better than the RCA-based partition ( $\triangle \mathrm{AIC}=-1,039.76$ and $\triangle \mathrm{BIC}=-224.01$ ). The multiple-groups analysis thus suggests that CCA offers a better description of the heterogeneity present in this data than does RCA.

\section{Discussion}

In this article, I introduced CCA, a technique that builds on Goldberg's (2011) RCA methodology. Both methods aim to partition a survey population into groups of respondents who arrange their tastes according to shared cultural schemas. RCA uses an eponymous relationality measure to quantify the extent to which two respondents appear to use such a "shared schema" - a central concept that has lacked a clear definition. By formalizing and making explicit the intuitions about such schemas implicit in Goldberg's work, I was able to substantially advance the clarity of this approach. Furthermore, my formalization showed that these kinds of shared schemas should manifest themselves in linear dependencies between pairs of response vectors-the same measurement task for which Pearson's correlation has long been an established solution. When I applied Pearson's correlation to the same example that Goldberg (2011) used to introduce relationality, I found that correlation yielded substantially more accurate results. I thus proposed CCA as a correlation-based alternative to RCA. 
I then used simulations to generalize and broaden the comparison between the two methods. In the first 10,000 simulations, I generated random taste schemas and then used randomly chosen noisy linear transformations to simulate respondents who follow these schemas. I measured the ability of both methods to correctly determine which sets of respondents were produced from the same schema and which were produced from different ones. I found that the accuracy of correlationbased CCA remained reliably higher than that of relationality-based RCA across the full range of simulation parameters. I also demonstrated that RCA relies on a strong distributional assumption. When the two methods produced different results, the odds that CCA was more accurate than RCA were 17:1 in runs that obeyed RCA's assumption, and they rose to $24: 1$ in the runs that violated it.

Because of the theoretical uncertainty surrounding schematic cognition and because Goldberg meant for RCA to capture nonlinear dependencies between tastes, I also investigated the performance of both methods when the model of schematic similarity as linearity was violated. I used simulations to examine six broad ways that schematic transformations could deviate from the theorized model. In the first three sets of simulations, I examined how the algorithms would perform if some basic linear operation (scaling, inversion, or shift) did not take place. In the second three sets, I examined performance in the presence of polynomial schematic transformations, independent subschemas, and multiway interactions between genre preferences. Even though these simulations violated the assumptions of my model, CCA remained consistently more accurate than RCA throughout the six sets of simulations, which indicated that relationality was not better than correlation at detecting any of these alternative patterns of schematic similarity. Overall, I found no evidence of any use cases in which RCA would be methodologically preferable over CCA.

I concluded with a reanalysis of the 1993 GSS musical tastes module previously analyzed by Goldberg (2011). Whereas RCA had partitioned this population into three classes, CCA partitioned it into four. Two of the four classes resembled those found by RCA, but the other two did not. This confirmed that the two methods can yield substantively different conclusions in empirical settings. I then examined their partitions of the GSS population with a multiple-groups analysis in SEM, which indicated that CCA's results yielded far better model fit to the GSS data than did RCA's.

In the above analyses, I found no evidence of any settings in which RCA would be methodologically justified over CCA. I am also not presently aware of any compelling theoretical reasons that could suggest the use of RCA over CCA. Although the theoretical justifications behind RCA and CCA have drawn on different rhetorical languages, these linguistic differences are not reflected in the actual substance of the two methods. One such linguistic difference that deserves special emphasis concerns the "relational" nature of the methods. Although CCA does not use Goldberg's relationality measure, CCA remains as relational a technique as RCA in both senses that Goldberg (2011) uses that term. First, both methods "simultaneously examine the relationships between variables and individuals" (Goldberg 2011:1404) by constructing networks of schematic similarities between respondents and then using these to partition them into classes. And second, both methods consider "the 
relationships between [respondents'] positions on a variety of issues that make up a certain social domain" (Goldberg 2011:1402) rather than examining these positions separately. Indeed, it appears appropriate that Galton (1888) originally referred to the measure he invented as "co-relation" rather than "correlation" — an alternative orthography that would render CCA as "Co-Relational Class Analysis" and thus would highlight CCA's equally relational nature.

\section{Limitations and Future Directions}

To highlight further avenues for methodological improvement, it is useful to consider RCA and CCA as two instances of a more general theoretical and methodological framework of schematic class analysis. This framework begins with three theoretical propositions:

1. Latent shared cultural schemas specify relative evaluations of tastes vis-à-vis each other in a cultural domain.

2. Individuals' taste patterns are manifestations of such schemas and are created from these schemas via one of a finite set of schematic transformations.

3. The individuals who created their taste patterns from the same schema make up a schematic class.

Then, methodologically:

4. The extent to which two respondents $i$ and $j$ appear to follow the same latent schema can be measured via some schematic similarity measure $S_{i j}$, which yields a similarity matrix $S$ when applied to all pairs of respondents.

5. Finally, it is possible to partition the matrix $S$ into zones of greater and lesser similarity via a partitioning method $(S)$, thus yielding an estimate of schematic classes present in the data.

6. These can then be used to estimate the contents of the shared cultural schemas.

Building on the intuitions behind Goldberg's (2011) work, I proposed here that shared cultural schemas (1) can be formalized as vectors of real numbers and schematic transformations (2) by linear functions. I therefore proposed to use Pearson's correlation as schematic similarity measure $S_{i j}(4)$. Like Goldberg, I employed modularity maximization for the partitioning method (5), which yields a partition of the survey population into schematic classes (3). I then showed how multiple-groups analysis can be used to quantify the fit of such a partition.

A theoretical argument for a different approach to detecting shared schemas would need to provide alternative answers to points (1) through (3). That is, an alternative model would need to either specify a different way that shared schemas can be captured (e.g., matrixes, functions, etc.), a different way that a schema can manifest itself in individuals' responses (i.e., other than through inversion, scaling, and shift), or offer an alternative conception of schematic class membership. Such 
theoretical challenges would then require different methodological answers to points (4) through (6).

To maximize analytical clarity, correlation can be used as a starting point for many such alterations. In the 129 years since Galton proposed an index of "corelation," a substantial number of other measures of correlation, such as Spearman's $\rho$ or point-biserial correlation, have been developed to capture this relationship under different special cases of data (Rodgers and Nicewander 1988). Many of these can be used as "drop-in replacements" for Pearson's correlation, thus making CCA easily adaptable to situations for which specialized alternatives to correlation already exist.

Moreover, the simple algebraic form of Pearson's $r$ makes it possible to derive custom correlation coefficients for many other potential situations. For example earlier in this article I examined three sublinear scenarios in which the basic linear schematic transformations of inversion, scaling, or shift were theorized not to occur. In Appendix $C$ of the online supplement, I demonstrate how Pearson's $r$ can be modified to yield three new indexes $\left(r_{\sim}, r_{\times}\right.$, and $\left.r_{+}\right)$, each of which makes the coefficient account for these theoretical changes.

Data sets. Other avenues for methodological development lie in adapting schematic class analysis to new types of data, such as the data sets from review websites like Yelp.com, Epinions.com, and Amazon.com that individuals use to publicly share their ratings of businesses, services, or products. Such data, which are private but regularly made available to researchers, greatly exceed traditional survey data in scale and richness of detail and thus provide important new opportunities for students of culture. Because Pearson's correlation is easy to compute efficiently and fast modularity-maximization algorithms are available, scaling CCA to a large $N$ is straightforward.

Schematic classes. Although this article has formally defined the concept of shared cultural schemas, the concept of schematic classes remains a second black box to be opened. How far can an individual's responses deviate from a schema for him or her to still count as a member of a schematic class, and how similar do two schemas have to be to actually count as one and the same schema? The answers to these questions fall to the partitioning method $\Phi(S)$. Presently, CCA (like RCA) uses modularity maximization as its partitioning method. As the simulations in this article demonstrated, it does its task with acceptable accuracy. But, although modularity maximization is among the most widely used network-analytic techniques across many disciplines (e.g., Porter, Onnela, and Mucha 2009; Shwed and Bearman 2010; Neal 2014), its use may implicitly introduce undesired theoretical assumptions. A substantial body of literature notes that modularity maximization suffers from a resolution limit that biases it against detecting both very small and very large modules in many empirical settings, joining even very different modules together when they are too small in proportion to the whole network and conversely breaking apart modules that are too large (Fortunato and Barthélemy 2007; Lancichinetti and Fortunato 2011; for a thorough overview, see Good, de Montjoye, and Clauset 2010).

By relying on modularity maximization, RCA and CCA may thus unintentionally introduce an assumption that each member of the survey population belongs to one of a moderate number of schematic classes and produce misleading results 
when this is not the case-for example, in situations in which many respondents follow their own idiosyncratic response patterns or in which the whole survey population belongs to a single schematic class. Future methodological work should develop a theoretically informed partitioning method $\Phi(S)$ that avoids these biases. ${ }^{28}$ Before this is done, RCA and CCA results should be examined to verify that they indeed contain a significant amount of heterogeneity, much as the results of other methods are tested for statistical significance or goodness of fit. In the GSS case study above, I've shown how this can be accomplished via a multiple-groups analysis in SEM.

\section{Conclusion}

In this article, I demonstrated that Pearson's correlation can be used to accurately measure schematic similarity between survey responses. Across the broad range of possible theoretical scenarios I examined, correlation-based CCA proved to be reliably more accurate at partitioning survey populations by shared cultural schemas than did relationality-based RCA. The switch from relationality to correlation brings a number of further benefits. Relationality is substantially more computationally costly to calculate than correlation and also requires bias correction and extensive bootstrapping for significance testing. Correlation obviates the need for these further steps. This leaves an algorithm that is clear, fast, and easy to implement (see Appendix A of the online supplement). It also clarifies and standardizes the method, thus placing it in closer conversation with other methodological work. Future improvements to CCA can draw insights from these existing literatures, thus helping further advance the methodological project that began with RCA.

\section{Notes}

1 In Martin's (2002) terminology, such schemas thus underlie the "tightness" rather than the "consensus" of a system of attitudes. If one imagines attitudes as an abstract space in which each dimension represents a like or dislike of a given musical genre, such schemas would specify an axis or plane along which culturally valid tastes can be arranged rather than a specific point in space at which tastes should be located.

2 Goldberg described cultural schemas as "complex structures of mental representation...that are built up incrementally through interaction with the environment [that] embody our taken-for-granted assumptions about the world," and further stated that "schemas are not clear sets of behavioral rules but rather implicit recognition procedures that emerge from intricate associational links among salient aspects of our cognitively represented experiences" (2011:1401). This description provides some intuitions about the functional role schemas play in culture and cognition. However, it is not the clear definition needed to assess whether relationality can accurately measure such schemas.

3 Formally, Goldberg (2011) defined the relationality between two respondents $i$ and $j$ to equal $R_{i j}=\frac{2}{K(K-1)} \sum_{k=1}^{K-1} \sum_{l=k+1}^{K}\left(\lambda_{i j}^{k l} * \delta_{i j}^{k l}\right)$, where $\delta_{i j}^{k l}=1-|| X_{i}^{k l}|-| X_{j}^{k l}||, X_{i}^{k l}=$ $X_{i}^{k}-X_{i}^{l}$ is the difference between the values of the variables $k$ and $l$ for respondent $i$, and $\lambda_{i j}^{k l}=1$ if $X_{i}^{k l}$ and $X_{j}^{k l}$ have the same sign or are both zero, and $\lambda_{i j}^{k l}=-1$ otherwise. 
4 Similarly, multiplying the timing sequence that makes up a melody's tempo by the same constant also keeps the melody recognizably the same (Radocy and Boyle 2012).

5 Goldberg (2011) reported some simulation tests of RCA's accuracy in his online Appendix B. However, his simulations consist entirely of individual taste vectors $(Y=X)$ and their exact inverses $(\mathrm{Y}=-\mathrm{X})$, plus noise. He reports an RCA accuracy of 100 percent in test cases without noise and 97.8 percent with noise. But these simulations are too easy: they cover only one of the three kinds of schematic transformation and miss $Y=k X$ and $\mathrm{Y}=\mathrm{X}+\mathrm{b}$. They also grant RCA's strong assumption that inversion probability equals 50 percent (see "Distributional Assumptions" below). To test performance under the full range of schematic similarity patterns in the motivating example, simulations would need to cover a substantially broader variety of scenarios. The simulations I report in the following sections cover this full range. On these more realistic tests, neither RCA nor CCA can reach such a high accuracy.

6 Respondents are assigned to classes based on the absolute values of the relationalities $\left|R_{i j}\right|$, so I plotted the values $\left|R_{i j}\right|$ instead of $R_{i j}$ to enable easier visual comparison of their magnitudes. I also omitted the Euclidean distances, which are not relevant to the present discussion. These changes make the limited dynamic range of relationality values more visually apparent. Because Goldberg does not present the numerical relationality scores for this example, I reproduced these values by measuring the bar lengths in his figure.

7 When I ran RCA with default parameters, it partitioned the population into 800 separate classes, thus assigning even identical rows to different classes. This obviously faulty solution appears to be due to the pseudosignificance testing RCA uses to filter weak relationalities, which is based on strong assumptions about how relationalities are distributed in the data. Disabling it produced the substantially more realistic solution I report above. (As I discuss below, this filter appears to generally decrease the average accuracy of RCA.)

8 Because of its complexity, I have thus far omitted discussion of the bias-adjustment procedure that RCA performs on the relationality scores before taking their absolute value. I will return to this introductory example later in the article to describe this procedure in due detail. In particular, I will show that the bias-reduction procedure relies on strong distributional assumptions that may be violated in much empirical data and can cause substantial problems when these assumptions do not hold. See "Distributional Assumptions" section below. Please also note that the RCA software did not offer an option to disable the bias-adjustment step. All the RCA analyses I report in this article thus include this bias adjustment.

9 For example, the schema shared by A, B, and C in Figure 1 can be specified as $\rho=$ $(0,0,0,1,1,-1,-1)$, so that $A=\rho+4, B=\rho+2$, and $C=-2 \rho+3$. Thus, the schemas are themselves defined up to a linear transformation.

10 Because of an apparent bug, the RCA software repeatedly crashed for 69 (1.3 percent) of these simulated data sets and produced no results. I excluded these cases from the analysis.

11 In spite of its popularity, this measure may at times produce unexpected results. In particular, NMI can be surprisingly forgiving of solutions that return too many classes: e.g., if the true data contain four classes, we would intuitively consider a 100-class solution to be almost completely wrong; however, NMI may still assign it an accuracy score above 0.5 . In supplementary analyses, I found that RCA returned such many-class solutions much more frequently than CCA; thus, any potential bias introduced by this measurement property appears to be conservative with regard to my argument here. I 
am indebted to John Levi Martin for alerting me to potential unexpected behaviors of this measure.

12 RCA software contains a user-configurable filtering step based on pseudosignificance testing, in which weak relationalities are dropped before partitioning to reduce potential noise. I examined how filtering affected RCA's performance using 250 simulation runs. Filtering increased accuracy in 56 percent of the cases but decreased it in 43 percent. However, the average decrease $(-0.33)$ was three times greater than the average increase (0.11). Overall, disabling the filter substantially raised RCA's median accuracy from 0.56 when enabled to 0.69 when disabled. Additionally, in 10 percent of the cases with filtering, RCA encountered an error and yielded no solution at all (as compared with 1 percent without filtering). Thus, to increase RCA's accuracy and avoid potential bias from substantial missing results, I disabled the filter for all the simulations reported in this article.

13 Those rare respondents with a variance of absolute zero require special treatment. See Appendix D of the online supplement for a detailed discussion.

14 It can also hold in a number of degenerate or improbable cases-for example, when there are roughly as many taste schemas as there are respondents.

15 See Appendix B of the online supplement ("Random Inversion Probability") for details of this procedure.

16 For a point of comparison, note that Pearson's correlation is only guaranteed to be unbiased and asymptotically efficient if the vectors are bivariate normal in distribution. To examine whether this is the case with my primary simulations, I applied the Royston $\mathrm{H}$ test for multivariate normality (Royston 1983) to 425 data sets randomly generated by the full linear simulation procedure from Appendix B of the online supplement. In each one of the 425 data sets, the hypothesis of multivariate normality was rejected at $\mathrm{p}<0.0001$. I then partitioned each simulated data set by schematic class membership so that respondents in each resulting data set would come from exactly one schematic class. In each one of these 1,728 single-class data sets, the hypothesis of multivariate normality was again rejected at $\mathrm{p}<0.0001$. The data produced by my linear simulation procedure thus violate correlation's bivariate normality assumption. Note that CCA was nonetheless able to analyze many of the simulated linear data sets with near-perfect accuracy.

17 Among other reasons, such differences in accuracy may arise if two measures have different amounts of sensitivity to inversion, scaling, and shift. Ceteris paribus, the measure that is more likely to identify that $X$ and its noisy inverted copy $Y=-1 * X+\epsilon$ follow the same schema, would probably yield a more accurate solution in the linear simulations examined above. However, the same property would then make it more likely to yield false positives in the "no inversion" scenario, in which two patterns that appear to be inverses of one another would actually be produced by two different schemas.

18 I described these operations in terms of individual action largely for the sake of readability. The actual sociocognitive processes of schematic transformation could well be supraindividual.

19 Any such examination would instead begin with an alternative model of how such schemas are represented and transformed (see "Limitations and Future Directions" section below).

20 One example of a polynomial transformation that does have an interpretation is a "center versus extremes" transformation that retains the extremity of the tastes in the 
schema but ignores the valence, thus treating "like a lot" and "dislike a lot" as equivalent (e.g., $X=\rho^{2},-10 \leq \rho \leq 10$ ).

21 See Procedure 3 in Appendix B of the online supplement for details.

22 Consider a situation in which there are three schemas $(a, b$, and $c)$, each of which can describe any musical genre. Then imagine that there are four groups of respondents: Group 1 uses $a$ to arrange all of its tastes; Group 2 uses $a$ to arrange tastes for popular genres only and $b$ for the remaining specialized genres; Group 3 instead uses $a$ for the specialized genres and $b$ for the popular ones; and Group 4 uses $c$ to evaluate jazz and rap, while using $a$ for all the remaining genres. It is not possible to partition this population in such a way that each respondent is assigned to a single class that corresponds to a single shared schema.

23 That is, if $a, b, c$, and $d$ are subschemas, and group $X$ uses the combination $(a, b)$ to arrange its tastes whereas group $Y$ uses combination $(c, d)$, we must assume that there is no group $Z$ that uses any other combination of these subschemas- $(a, c),(b, c),(a, d)$, or $(b, d)$. We must also assume that $a$ defines no genres defined by $b$, and $c$ defines no genres by $d$.

24 See Procedure 4 in Appendix B of the online supplement for details..

25 A population of respondents who use transformations such as these would be introducing cultural logics that are not part of the schema. Consider, for example, a schema like $S=\left[S_{\text {rock }}=1, S_{\text {rap }}=1, S_{\text {classical }}=4, S_{\text {opera }}=4\right]$ on a scale from $1=$ like to $4=$ dislike and a respondent $Z$ using the transformations $Z_{\text {rock }}=\left(S_{\text {rock }}^{2} * S_{\text {opera }} * S_{\text {classical }}\right)^{.25}=2$ and $Z_{\text {classical }}=\left(S_{\text {classical }}^{2} * S_{\text {rock }} * S_{\text {rap }}\right)^{.25}=2$. Although the Lowbrow-Highbrow schema $S$ placed rock in opposition to classical, and $Z$ produced her tastes without any error, she nonetheless ended up with an omnivorous taste pattern.

26 The general functional form of the transformation that produces a respondent's taste $R_{k}$ in interaction block $\left\{R_{k}, R_{m}, R_{n}\right\}$ from schema $\rho$ is the weighted geometric mean $R_{k}=g\left(\rho_{k}, \rho_{m}, \rho_{n}\right)=\sqrt[a+2]{\rho_{k}^{a} \rho_{m} \rho_{n}}$. I attached weight a to the focal element $\rho_{k}$ because without weights, the respondent's tastes for all genres in the interaction block would be identical: $\sqrt[3]{\rho_{k} \rho_{m} \rho_{n}}=\sqrt[3]{\rho_{m} \rho_{k} \rho_{n}}=\sqrt[3]{\rho_{n} \rho_{k} \rho_{m}}$. I overrode this unintuitive behavior in roughly 75 percent of the runs by randomly drawing $a \sim U\{1,2,3,4\}$. When $a>1, \rho_{k}$ has a stronger effect on $R_{k}$ than do $\rho_{m}$ or $\rho_{n}$. See Procedure 5 in Appendix B of the online supplement for the detailed simulation procedure.

27 The parentheses around "but" in "Anything (but) Country" are meant to signify that the class could equally be named "Anything but Country" or "Anything Country"—that is, that the logic opposes "any music that isn't country" to "any music that is country." The same logic applies to "Anything (but) Heavy Metal."

28 For an example of a partitioning technique with a domain-specific fix to problems stemming from modularity's resolution limit, see Sohn et al. (2011).

\section{References}

Baldassarri, Delia, and Amir Goldberg. 2014. "Neither Ideologues, nor Agnostics: Alternative Voters' Belief System in an Age of Partisan Politics." American Journal of Sociology. https : //doi.org/10.1086/676042

Bollen, Kenneth A. 1989. Structural Equations with Latent Variables. New York: John Wiley \& Sons. https://doi.org/10.1002/9781118619179 
Bourdieu, Pierre. 1987. Distinction: A Social Critique of the Judgement of Taste. Harvard University Press.

Boutyline, Andrei, and Stephen Vaisey. 2017. "Belief Network Analysis: A Relational Approach to Understanding the Structure of Attitudes." American Journal of Sociology 122(5):1371-1447. https://doi.org/10.1086/691274

Bryson, Bethany. 1996. “Anything But Heavy Metal: Symbolic Exclusion and Musical Dislikes." American Sociological Review 61(5):884-99. https://doi .org/10.2307/2096459

Cerulo, Karen A. 1993. "Symbols and the World System: National Anthems and Flags." Sociological Forum 8(2):243-71. https: //doi.org/10.1007/BF01115492

Daenekindt, Stijn. 2017. “On the structure of dispositions. Transposability of and oppositions between aesthetic dispositions." Poetics 62: 43-52. https://doi .org/10.1016/j . poetic . 2017.01 .004 .

Danon, Leon, Albert Díaz-Guilera, Jordi Duch, and Alex Arenas. 2005. “Comparing Community Structure Identification." Journal of Statistical Mechanics: Theory and Experiment 2005(09):P09008.

DiMaggio, Paul, and Amir Goldberg. 2010. "Searching for Homo Economicus: Variation in the Structure of Americans' Moral Evaluations of Markets." Atlanta, GA.

Emirbayer, Mustafa. 1997. "Manifesto for a Relational Sociology." American Journal of Sociology 103(2):281-317. https : //doi .org/10.1086/231209

Fararo, Thomas J., and John Skvoretz. 1987. "Unification Research Programs: Integrating Two Structural Theories." American Journal of Sociology 92(5):1183-1209. https://doi . org/10.1086/228632

Fortunato, Santo, and Marc Barthélemy. 2007. "Resolution Limit in Community Detection." Proceedings of the National Academy of Sciences 104(1):36-41. https : //doi .org/10.1073/ pnas. 0605965104

Galton, Francis. 1888. "Co-Relations and Their Measurement, Chiefly from Anthropometric Data." Proceedings of the Royal Society of London 45:135-45. https://doi .org/10.1098/ rspl.1888.0082

Goldberg, Amir. 2011. "Mapping Shared Understandings Using Relational Class Analysis: The Case of the Cultural Omnivore Reexamined." American Journal of Sociology 116(5):1397-1436.

Goldberg, Amir, and Jinjian Zhai. 2013. RCA R Package. Retrieved from CRAN repository. https://doi .org/10.1086/657976

Good, Benjamin H., Yves-Alexandre de Montjoye, and Aaron Clauset. 2010. "Performance of Modularity Maximization in Practical Contexts." Physical Review E 81(5):046106. https : //doi.org/10.1103/PhysRevE.81.046106

Gregorich, Steven E. 2006. “Do Self-Report Instruments Allow Meaningful Comparisons Across Diverse Population Groups? Testing Measurement Invariance Using the Confirmatory Factor Analysis Framework." Medical Care 44(11 Suppl 3):S78-94. https://doi.org/10.1097/01.mlr.0000245454.12228.8f

Hummel, John. 2013. “Object Recognition.” In The Oxford Handbook of Cognitive Psychology. Oxford University Press, USA. https://doi .org/10.1093/oxfordhb/9780195376746. 013.0003

Kool, Wouter, Joseph T. McGuire, Zev B. Rosen, and Matthew M. Botvinick. 2010. “Decision Making and the Avoidance of Cognitive Demand." Journal of Experimental Psychology: General 139(4):665-82. https://doi.org/10.1037/a0020198 
Lancichinetti, Andrea, and Santo Fortunato. 2011. "Limits of Modularity Maximization in Community Detection." Physical Review E 84(6):066122. https : //doi . org/10.1103/ PhysRevE.84.066122

Lancichinetti, Andrea, Santo Fortunato, and János Kertész. 2009. “Detecting the Overlapping and Hierarchical Community Structure in Complex Networks." New Journal of Physics 11(3):033015. https://doi.org/10.1088/1367-2630/11/3/033015

Lévi-Strauss, Claude. 1963. Structural Anthropology. Basic Books.

MacCallum, Robert, and James Austin. 2000. "Applications of Structural Equation Modeling in Psychological Research." Annual Review of Psychology 51(1):201-26. https : //doi .org/ 10.1146/annurev.psych.51.1.201

Manning, Christopher D., Prabhakar Raghavan, and Hinrich Schütze. 2008. Introduction to Information Retrieval. New York: Cambridge University Press.

Martin, John Levi. 2002. "Power, Authority, and the Constraint of Belief Systems." American Journal of Sociology 107(4):861-904. https : //doi .org/10.1086/343192

McPherson, Miller. 2004. "A Blau Space Primer: Prolegomenon to an Ecology of Affiliation." Industrial and Corporate Change 13(1):263-80. https://doi .org/10.1093/icc/13.1.263

McPherson, Miller, Lynn Smith-Lovin, and James M. Cook. 2001. "Birds of a Feather: Homophily in Social Networks." Annual Review of Sociology 27(1):415-44. https ://doi . org/10.1146/annurev.soc.27.1.415

Miranda, Shaila, Jama Summers, and Inchan Kim. 2012. "Visions of Social Media: Surfacing Schemas from Firms' Informational Engagements." ICIS 2012 Proceedings.

Mohr, John W. 1998. "Measuring Meaning Structures." Annual Review of Sociology 24:345-70. https://doi.org/10.1146/annurev.soc.24.1.345

Mohr, John W., and Craig Rawlings. 2012. "Four Ways to Measure Culture: Social Science, Hermeneutics and the Cultural Turn." In The Oxford Handbook of Cultural Sociology. Oxford University Press, USA.

Neal, Zachary. 2014. "The Devil Is in the Details: Differences in Air Traffic Networks by Scale, Species, and Season." Social Networks 38:63-73. https://doi.org/10.1016/j.socnet. 2014.03 .003

Newman, Mark E. J. 2006. "Modularity and Community Structure in Networks." Proceedings of the National Academy of Sciences 103(23):8577-82. https://doi.org/10.1073/pnas. 0601602103

Peterson, Richard A. 1997. "The Rise and Fall of Highbrow Snobbery as a Status Marker." Poetics 25(2-3):75-92.

Peterson, Richard A. 2005. "Problems in Comparative Research: The Example of Omnivorousness." Poetics 33(5-6):257-82.

Peterson, Richard A., and Roger M. Kern. 1996. “Changing Highbrow Taste: From Snob to Omnivore." American Sociological Review 61(5):900-907. https : //doi .org/10 . 2307/ 2096460

Porter, Mason, Jukka-Pekka Onnela, and Peter Mucha. 2009. "Communities in Networks." Notices of the American Mathematical Society 56(9).

Radocy, Rudolf, and J. David Boyle. 2012. Psychological Foundations of Musical Behavior. 5th ed. Charles C Thomas Publisher.

Ritzer, George, and Jeff Stepnisky. 2013. Sociological Theory. 9th edition. New York, NY: McGraw-Hill. 
Rodgers, Joseph Lee, and W. Alan Nicewander. 1988. "Thirteen Ways to Look at the Correlation Coefficient." The American Statistician 42(1):59-66. https://doi.org/10. $2307 / 2685263$

Royston, J. P. 1983. "Some Techniques for Assessing Multivariate Normality Based on the Shapiro- Wilk W." Journal of the Royal Statistical Society. Series C (Applied Statistics) 32(2):121-33.

de Saussure, Ferdinand. 1916. Course in General Linguistics. Columbia University Press.

Shwed, Uri, and Peter S. Bearman. 2010. “The Temporal Structure of Scientific Consensus Formation." American Sociological Review 75(6):817-40. https://doi .org/10.1177/ 0003122410388488

Sohn, Yunkyu, Myung-Kyu Choi, Yong-Yeol Ahn, Junho Lee, and Jaeseung Jeong. 2011. "Topological Cluster Analysis Reveals the Systemic Organization of the Caenorhabditis Elegans Connectome." PLoS Comput Biol 7(5):e1001139. https://doi.org/10.1371/ journal.pcbi.1001139

Stockburger, David. 2011. Introductory Statistics: Concepts, Models, and Applications (2 ${ }^{\text {nd }}$ ed.) Atomic Dog Publishing.

Tampubolon, Gindo. 2008. "Revisiting Omnivores in America circa 1990s: The Exclusiveness of Omnivores?" Poetics 36(2-3):243-64.rence

Taylor, Shelley E. 1981. “The Interface of Cognitive and Social Psychology.” Cognition, Social Behavior, and the Environment 189-211.

Ullman, Shimon. 1989. "Aligning Pictorial Descriptions: An Approach to Object Recognition." Cognition 32(3):193-254. https : //doi .org/10.1016/0010-0277 (89)90036-X

Wu, Angela Xiao. 2014. "Ideological Polarization Over a China-as-Superpower Mindset: An Exploratory Charting of Belief Systems Among Chinese Internet Users, 2008-2011." International Journal of Communication 8: 2243-2272.

Acknowledgements: This research was supported in part by fellowships from National Science Foundation Graduate Research Fellowship Program and Interdisciplinary Graduate Education and Research Traineeship Program. I thank Ronald Breiger, Neil Fligstein, John Flournoy, Amir Goldberg, Monica Lee, Valden Kamph, James Kitts, Fabiana Silva, Matthew Stimpson, Stephen Vaisey, Robb Willer, and the participants of the Berkeley Mathematical, Analytical, and Experimental Sociology workshop for feedback on the article. I am also grateful to Amir Goldberg for generously discussing RCA and making its software implementation available online. Direct all correspondence to Andrei Boutyline at Department of Sociology, 410 Barrows Hall, University of California, Berkeley, CA 94720. E-mail: boutyline@berkeley.edu

Andrei Boutyline : Department of Sociology, University of California, Berkeley. E-mail: boutyline@berkeley.edu. 